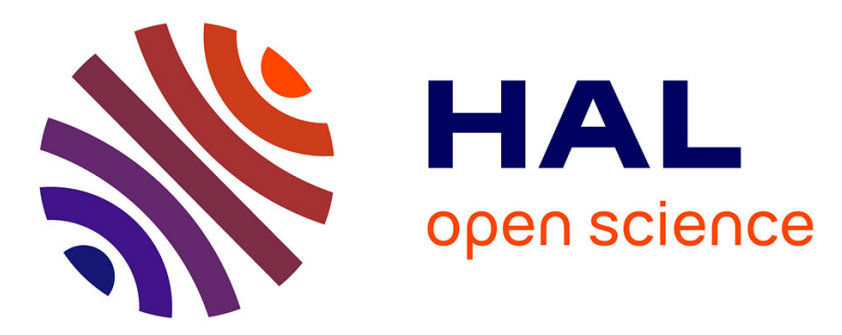

\title{
Hybrid-dimensional modelling of two-phase flow through fractured porous media with enhanced matrix fracture transmission conditions
}

Konstantin Brenner, Julian Hennicker, Roland Masson, Pierre Samier

\section{- To cite this version:}

Konstantin Brenner, Julian Hennicker, Roland Masson, Pierre Samier. Hybrid-dimensional modelling of two-phase flow through fractured porous media with enhanced matrix fracture transmission conditions. Journal of Computational Physics, 2018, 10.1016/j.jcp.2017.12.003 . hal-01518930v2

\section{HAL Id: hal-01518930 \\ https://hal.science/hal-01518930v2}

Submitted on 9 Nov 2017

HAL is a multi-disciplinary open access archive for the deposit and dissemination of scientific research documents, whether they are published or not. The documents may come from teaching and research institutions in France or abroad, or from public or private research centers.
L'archive ouverte pluridisciplinaire HAL, est destinée au dépôt et à la diffusion de documents scientifiques de niveau recherche, publiés ou non, émanant des établissements d'enseignement et de recherche français ou étrangers, des laboratoires publics ou privés. 


\title{
Hybrid-dimensional modelling of two-phase flow through fractured porous media with enhanced matrix fracture transmission conditions
}

\author{
K. Brenner*, J. Hennicker*†, R. Masson*, P. Samier ${ }^{\dagger}$
}

November 9, 2017

\begin{abstract}
In this work, we extend, to two-phase flow, the single-phase Darcy flow model proposed in [26], [12] in which the $(d-1)$-dimensional flow in the fractures is coupled with the $d$-dimensional flow in the matrix. Three types of so called hybrid-dimensional two-phase Darcy flow models are proposed. They all account for fractures acting either as drains or as barriers, since they allow pressure jumps at the matrix-fracture interfaces. The models also permit to treat gravity dominated flow as well as discontinuous capillary pressure at the material interfaces. The three models differ by their transmission conditions at matrix fracture interfaces: while the first model accounts for the nonlinear two-phase Darcy flux conservations, the second and third ones are based on the linear single phase Darcy flux conservations combined with different approximations of the mobilities. We adapt the Vertex Approximate Gradient (VAG) scheme to this problem, in order to account for anisotropy and heterogeneity aspects as well as for applicability on general meshes. Several test cases are presented to compare our hybrid-dimensional models to the generic equi-dimensional model, in which fractures have the same dimension as the matrix, leading to deep insight about the quality of the proposed reduced models.
\end{abstract}

\section{Introduction}

This work is concerned with the modelling and the discretization of two-phase Darcy flows in fractured porous media, for which the fractures are represented as interfaces of codimension one. In this framework, the $(d-1)$-dimensional flow in the fractures is coupled with the $d$-dimensional flow in the matrix leading to the so called, hybrid-dimensional Darcy flow models. These models are derived from the so called equi-dimensional model, where fractures are represented as geological structures of equal dimension as the matrix, by averaging fracture quantities over the fracture width. We consider the case for which the pressure can be discontinuous at the matrix-fracture $(m f)$ interfaces in order to account for fractures acting either as drains or as barriers as described in [19, 26, 6, 12], contrary to the continuous pressure model described in $[3,11]$ developed for conductive fractures.

Our objective is to compare different models extending hybrid-dimensional discontinuous pressure models to two-phase Darcy flows. The comparison between models uses the equi-dimensional model to provide a reference solution. A rather simple 2D setting is considered to be able to compute this reference solution and make the comparison. The targeted hybrid-dimensional model should be able to account accurately for gravity effects inside the fractures, for discontinuous capillary pressure curves at $m f$ interfaces and for both drains and barriers. Most existing hybrid-dimensional two-phase Darcy flow models are based on the assumption of continuous phase pressures at the $m f$ interfaces assuming fractures acting as drains $[8,31,30,24,9,10]$. Another existing approach, accounting for both drains or barriers, amounts to eliminate the interfacial phase pressures using the linear single phase Darcy

\footnotetext{
*Laboratoire de Mathématiques J.A. Dieudonné, UMR 7351 CNRS, University Nice Sophia Antipolis, and team COFFEE, INRIA Sophia Antipolis Méditerranée, Parc Valrose 06108 Nice Cedex 02, France, \{konstantin.brenner, julian.hennicker, roland.masson\}@unice.fr

${ }^{\dagger}$ CSTJF, TOTAL S.A. - Avenue Larribau, 64018 Pau, France
} 
flux conservation equation at the $m f$ interfaces for each phase. It is usually combined with Two Point [25] or MultiPoint [35, 33, 1] cell centred finite volume schemes for which the interfacial unknowns can be easily eliminated when building the single phase Darcy flux transmissivities. It is important to notice that, in the case of fractures acting as drains, this approach provides basically the same solutions as the ones obtained by the continuous pressure model. These hybrid-dimensional models are here compared with a new hybrid-dimensional model which

- accounts for the $m f$ interfacial phase pressures in addition to the phase pressures averaged along the width of the fracture,

- models the two-phase Darcy normal fluxes in the fracture at each $m f$ interface using a Two Point Flux Approximation with phase based upwinding, gravity term and saturation jump,

- writes the nonlinear two-phase Darcy flux conservation equations at the $m f$ interface.

To our knowledge, this type of hybrid-dimensional two-phase Darcy flow model has not been studied before, except in $[27,2]$ where the authors use a global pressure formulation. In order to account more efficiently for discontinuous capillary pressures, our model uses the phase pressures as primary unknowns. This formulation is based on the inverse of the monotone graph extension of the capillary pressure curves and can be easily extended to general capillary pressure curves including vanishing capillary pressures in the fracture using a switch of variable formulation as described in [10] in the case of continuous pressure models. Our coupling conditions at the $m f$ interfaces also differ from the ones presented in $[27,2]$ in the sense that they incorporate an upwinding between the matrix and fracture mobilities and do not neglect the gravitational force. This upwinding is crucial in order to transport fluid from the matrix to the fractures and the gravitational force in the width of the fracture cannot be neglected for gravity dominated flows independently on the fracture width.

An important novelty of the proposed hybrid-dimensional model is that the saturations at the $m f$ interfaces are explicitly calculated, in addition to the mean saturations along the width of the fractures. All of the aforementioned models [8, 31, 30, 24, 9, 10, 25] lack in this supplementary information. They either have only the mean physical unknowns along the width of the fractures, which is the case for the hybrid-dimensional continuous pressure models, or they eliminate the interfacial pressures using the linear single phase flux conservation equations. Note again that this linear elimination provides basically the same results as continuous pressure models in the case of fractures acting as drains. The importance of preserving both the $m f$ interfacial and mean saturations becomes obvious in the test case section of this work: the influence on the solution of capillary or gravitational forces in normal direction within the fractures is far from being negligible, in general. The supplementary unknowns at the $m f$ interfaces enable the method presented in this paper to capture these effects. This is of course a very different situation than what occurs for a single phase flow possibly coupled with a linear transport tracer equation for which the pressure (and possibly tracer) continuity assumption at the $m f$ interfaces provides very accurate results in the case of fractures acting as drains.

The discretization of hybrid-dimensional Darcy flow models has been the object of several works. For a review of existing methods, we refer to [32, 34]. For single-phase Darcy flow, a cell-centered Finite Volume scheme using a Two Point Flux Approximation (TPFA) is proposed in [25, 19, 6] assuming the orthogonality of the mesh and isotropic permeability fields. Cell-centred Finite Volume schemes can be extended to general meshes and anisotropic permeability fields using MultiPoint Flux Approximations (MPFA) following [35, 33, 1]. Nevertheless, MPFA schemes can lack robustness on distorted meshes and for large anisotropies due to the non symmetry of the discretization. They are also very expensive compared to nodal discretizations on tetrahedral meshes. In [26], a Mixed Finite Element (MFE) method is proposed. More recently the Hybrid Finite Volume (HFV) scheme, introduced in [15], has been extended in [18] for the geometrically non-conforming discretization of two reduced fault models. Also a Mimetic Finite Difference (MFD) scheme is used in [7] in the matrix domain coupled with a TPFA scheme in the fracture network. Discretizations of the related reduced model [3] assuming a continuous pressure at the $m f$ interfaces have been proposed in [3] 
using a MFE method and in [11] using the HFV scheme and an extension of the Vertex Approximate Gradient (VAG) scheme introduced in [14]. Finally, the VAG and HFV schemes have been extended to the single-phase hybrid-dimensional discontinuous pressure model in [12]. Let us also mention two classes of so-called geometrically non-conforming discretizations, that handle non-matching fracture and matrix meshes: the Extended Finite Element Method [5, 20, 21] and the Embedded Discrete Fracture Method [29, 23].

For two-phase Darcy flow, the TPFA discretization is used in [25] with elimination of the $m f$ interface pressures when computing the Darcy flux transmissivities. In [24], the two-phase flow equations are solved in an IMPES framework, using a Mixed Hybrid Finite Element (MHFE) discretization for the pressure equation and a Discontinuous Galerkin discretization of the saturation equation. Either a zero flux or the pressure continuity are assumed at $m f$ interfaces. The paper also contains a review on the most common numerical approaches, when dealing with discrete fractures. The Hybrid Finite Volume discretization (HFV, see $[15,11]$ ) is extended in [22] to two-phase Darcy flow in fractured media with continuous pressure at $m f$ interfaces. These approaches are adapted to general meshes and anisotropy but require as many degrees of freedom as faces. An early paper to use a Control Volume Finite Element method (CVFE) for the discretization of hybrid-dimensional two-phase flow is [8] assuming pressure continuity at $m f$ interfaces. Still for continuous pressure models, a CVFE scheme is proposed in [31] that uses reconstruction operators for the saturations that depend on the rock characteristic capillary pressure curves. In this way, the saturation jumps (due to discontinuous capillary pressure) at the material interfaces are respected. A similar approach can be found in [30] combined with a switch of variable technique to account for highly contrasted $m f$ capillary pressures. However, the rigid choice of the control volumes (that are the dual cells) leads to the need of small matrix cells at the DFN neighbourhood, in order not to enlarge the drains artificially. In $[9,10]$, the VAG scheme is used to discretize the continuous pressure model, which is very flexible in the distribution of control volumes and hence circumvents this problem.

In this work, we present an adaptation of the VAG scheme

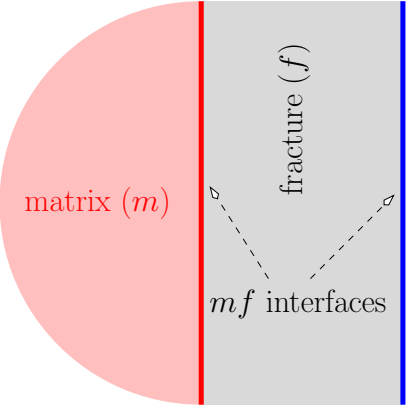

Figure 1: Nomenclature. to the hybrid-dimensional discontinuous pressure model using supplementary unknowns at the $m f$ interfaces to capture the pressure jumps as initially proposed in [12]. We choose a vertex based scheme, because it leads, compared to the cellcentered and face-centered approaches, to a smaller number of degrees of freedom when dealing with triangular or tetrahedral meshes, which in turn often arise when complex geometries have to be taken into account. Furthermore, the control volume version of the VAG scheme, presented here, allows to take into account saturation jumps (due to capillary pressure) at rock type interfaces including the $m f$ interfaces.

The outline of this work is as follows. The hybrid-dimensional two-phase flow models are provided in section 2. The discontinuous hybrid-dimensional model presented in section 2.2 is based on the two-phase Darcy flux conservation equations at the $m f$ interfaces (hybrid-dimensional $m f$ nonlinear model). At the discrete level, we propose a modification of this model in section 3.3, which still accounts for pressure discontinuities at the $m f$ interfaces, but provides linear matrix-fracture transmission conditions (hybrid-dimensional $m f$ linear model). For this latter model, two types of upwinding of the $m f$ fluxes are investigated for fluxes going out of the fracture. The first one is based on the matrix capillary pressure curve and capture the saturation jump (hybrid-dimensional $m f$ linear $m$ upwind model). The second one uses the fracture capillary pressure curve in the spirit of cell centred finite volume schemes [25] (hybrid-dimensional $m f$ linear $f$ upwind model).

Section 3 is devoted to the VAG discretization and provides a finite volume formulation of the models. In section 4, the hybrid and equi-dimensional models are compared on a linear tracer problem coupled to a single phase Darcy flow equation with analytical solutions. For such problem, the three 
hybrid-dimensional models exactly match. Our objective is to assess their accuracy as a function of the conductivity ratio between the fracture and the matrix. The effect of a normal diffusion term in the fracture for the equi-dimensional model is also investigated. The section 5 compares the three hybrid-dimensional models with the equi-dimensional model on a two-phase flow problem with different matrix and fracture permeabilities and capillary pressures. The last section 6 presents a 3D test case with anisotropic matrix permeability to assess the efficiency of the hybrid-dimensional $m f$ nonlinear model on a family of three tetrahedral meshes.

\section{Hybrid-dimensional Modelling of Flow in Fractured Porous Media}

Fractures and faults are geological structures with highly contrasted petro- and hydrological properties and a small thickness compared to the surrounding matrix. To derive the hybrid-dimensional model, we average the continuity and Darcy equations over the fracture width. The main objectives are to facilitate mesh generation and to decrease the number of degrees of freedom involved in the numerical resolution of the corresponding discrete problem.

\section{$2.1 \quad$ Discrete Fracture Network}

Let $\Omega$ denote a bounded domain of $\mathbb{R}^{d}, d=2,3$ assumed to be polyhedral for $d=3$ and polygonal for $d=2$. To fix ideas the dimension will be set to $d=3$ when it needs to be specified, for instance in the naming of the geometrical objects or for the space discretization in the next section. The adaptations to the case $d=2$ are straightforward.

Let $\bar{\Gamma}=\bigcup_{i \in I} \bar{\Gamma}_{i}$ and its interior $\Gamma=\bar{\Gamma} \backslash \partial \bar{\Gamma}$ denote the network of fractures $\Gamma_{i} \subset \Omega, i \in I$, which is a collection of planar polygonal simply connected open domains, such that each $\Gamma_{i}$ is included in a plane $\mathcal{P}_{i}$ of $\mathbb{R}^{d}$. Without loss of generality, we will assume that the angles of $\Gamma_{i}$ are strictly smaller than $2 \pi$, and that $\Gamma_{i} \cap \bar{\Gamma}_{j}=\emptyset$ for all $i \neq j$. For all $i \in I$, let us set $\Sigma_{i}=\partial \Gamma_{i}$, with $\mathbf{n}_{\Sigma_{i}}$ as unit vector in $\mathcal{P}_{i}$, normal to $\Sigma_{i}$ and outward to $\Gamma_{i}$. Further $\Sigma_{i, j}=\Sigma_{i} \cap \Sigma_{j}, j \in I \backslash\{i\}, \Sigma_{i, 0}=\Sigma_{i} \cap \partial \Omega$, $\Sigma_{i, N}=\Sigma_{i} \backslash\left(\bigcup_{j \in I \backslash\{i\}} \Sigma_{i, j} \cup \Sigma_{i, 0}\right), \Sigma=\bigcup_{(i, j) \in I \times I, i \neq j}\left(\Sigma_{i, j} \backslash \Sigma_{i, 0}\right)$ and $\Sigma_{0}=\bigcup_{i \in I} \Sigma_{i, 0}$.
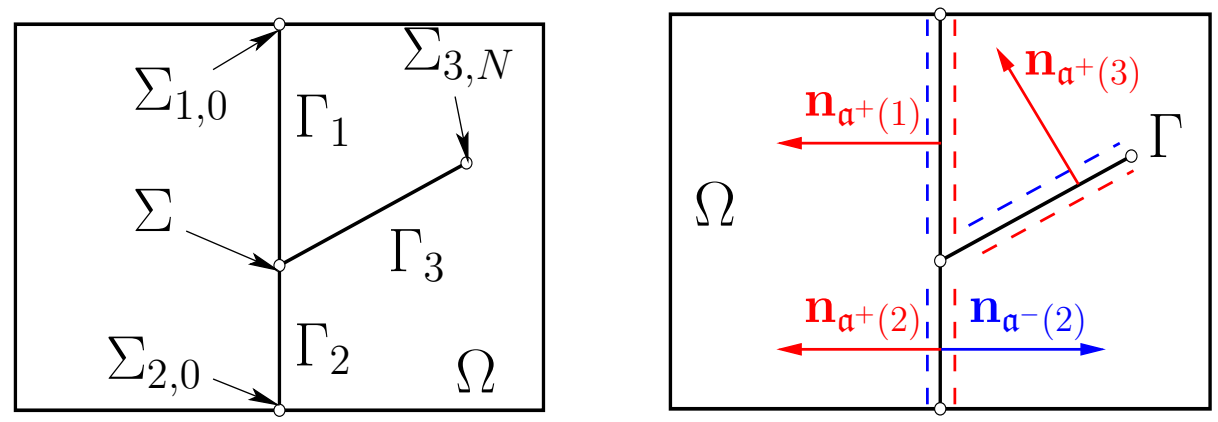

Figure 2: Example of a 2D domain $\Omega$ and 3 intersecting fractures $\Gamma_{i}, i=1,2,3$. We define the fracture plane orientations by $\mathfrak{a}^{ \pm}(i) \in \chi$ for $\Gamma_{i}, i \in I$.

We define the two unit normal vectors $n_{\mathfrak{a}^{ \pm}(i)}$ at each planar fracture $\Gamma_{i}$, such that $\mathbf{n}_{\mathfrak{a}^{+}(i)}+\mathbf{n}_{\mathfrak{a}^{-}(i)}=0$ (cf. figure 2). We define the set of indices $\chi=\left\{\mathfrak{a}^{+}(i), \mathfrak{a}^{-}(i) \mid i \in I\right\}$, such that $\# \chi=2 \# I$. For ease of notation, we use the convention $\Gamma_{\mathfrak{a}^{+}(i)}=\Gamma_{\mathfrak{a}^{-}(i)}=\Gamma_{i}$. Then, for $\mathfrak{a}=\mathfrak{a}^{ \pm}(i) \in \chi$, we can define the trace operator on $\Gamma_{\mathfrak{a}}$ :

$$
\gamma_{\mathfrak{a}}: H^{1}(\Omega \backslash \bar{\Gamma}) \rightarrow L^{2}\left(\Gamma_{\mathfrak{a}}\right),
$$

and the normal trace operator on $\Gamma_{\mathfrak{a}}$ outward to the side $\mathfrak{a}$ :

$$
\gamma_{\mathbf{n}_{\mathfrak{a}}}: H_{\operatorname{div}}(\Omega \backslash \bar{\Gamma}) \rightarrow \mathcal{D}^{\prime}\left(\Gamma_{\mathfrak{a}}\right),
$$


that satisfy $\gamma_{\mathfrak{a}}(h)=\gamma_{\Gamma_{i}}\left(h \uparrow_{\omega_{\mathfrak{a}}}\right)$ and $\gamma_{\mathbf{n}_{\mathfrak{a}}}(\mathbf{p})=\gamma_{\mathbf{n}, \Gamma_{i}}\left(\left.\mathbf{p}\right|_{\omega_{\mathfrak{a}}}\right)$, where $\omega_{\mathfrak{a}}=\left\{\mathbf{x} \in \Omega \mid(\mathbf{x}-\mathbf{y}) \cdot \mathbf{n}_{\mathfrak{a}}<0, \forall \mathbf{y} \in \Gamma_{i}\right\}$, and where $\gamma_{\Gamma_{i}}$ and $\gamma_{\mathbf{n}, \Gamma_{i}}$ are the usual trace and normal trace operators defined of $H^{1}\left(\Omega_{\mathfrak{a}}\right)$ and $H_{\text {div }}\left(\omega_{\mathfrak{a}}\right)$ respectively.

\subsection{Two-phase Darcy flow models}

Equi-dimensional model We consider the flow equations for incompressible, immiscible two-phase flow on a $d$-dimensional porous domain $\Omega$ containing a fracture $\Omega_{f}$ as illustrated in figure 3 . To simplify, we consider no sources or sinks.

$$
\begin{aligned}
\phi \partial_{t} S^{\alpha}(\mathbf{x}, p)+\operatorname{div}\left(\mathbf{q}^{\alpha}\right) & =0, \\
\mathbf{q}^{\alpha} & =-k^{\alpha}\left(\mathbf{x}, S^{\alpha}(\mathbf{x}, p)\right) \Lambda\left(\nabla u^{\alpha}-\rho^{\alpha} \mathbf{g}\right),
\end{aligned}
$$

with phase parameter $\alpha \in\{1,2\}$ ( 1 for a non wetting phase and 2 for a wetting phase), phase pressures $u^{\alpha}$, phase Darcy velocities $\mathbf{q}^{\alpha}$, capillary pressure $p$, phase saturations $S^{\alpha}$, permeability tensor $\Lambda$ (positive definite), porosity $\phi$, phase mobility $k^{\alpha}$ phase mass densities $\rho^{\alpha}$ gravitational vector field $\mathbf{g}$ and space coordinate $\mathbf{x} \in \Omega$. The system is closed by the equations

$$
p=u^{1}-u^{2}, \quad S^{1}(\mathbf{x}, p)+S^{2}(\mathbf{x}, p)=1 .
$$

Model (1) will be referred to as the equi-dimensional model, in the following.

Derivation of the hybrid-dimensional models We suppose that the matrix and the fracture network consist of a finite number of rocks, that define finite partitions of $\Omega \backslash \Omega_{f}$ and $\Omega_{f}$. To identify those rocks mathematically, we attribute a proper rock type $r t$ to each open set $\omega_{r t}$ of these partitions. Then, it is assumed that for each given rock type $r t$, the functions $k^{\alpha}(\mathbf{x},$.$) and S^{\alpha}(\mathbf{x},$.$) are independent$ on $\mathbf{x} \in \omega_{r t}$. Moreover, for each given rock type $r t$ and any $\mathbf{x} \in \omega_{r t}, S^{1}(\mathbf{x}, q) \in[0,1]$ for all $q \in \mathbb{R}$ and $S^{1}(\mathbf{x},$.$) is a non decreasing continuous function on \mathbb{R}$, and $k^{\alpha}(\mathbf{x},$.$) is a continuous, non-negative$ valued function on $[0,1]$, for $\alpha=1,2$.
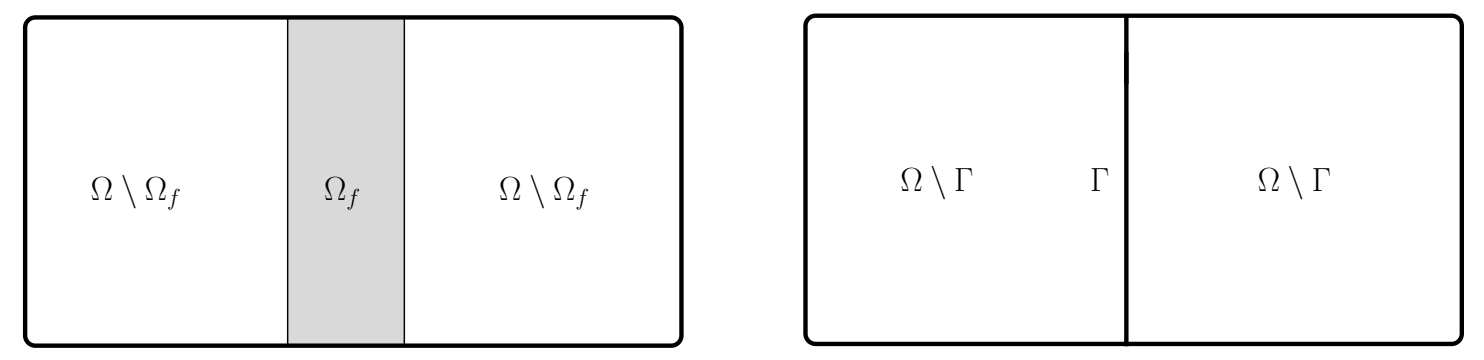

Figure 3: Geometries of the equi-dimensional model (left) and the hybrid-dimensional model (right) in the case of a single fracture dividing the matrix domain.

The fracture has the representation $\Omega_{f}=\left\{\mathbf{x} \in \Omega \mid \mathbf{x}=\mathbf{y}+r \mathbf{n}(\mathbf{y}), \mathbf{y} \in \Gamma, r \in\left(-\frac{d_{f}(\mathbf{y})}{2}, \frac{d_{f}(\mathbf{y})}{2}\right)\right\}$, where $\mathbf{n}$ is a unit normal vector and $d_{f}$ is the fracture width. Let us further introduce the orthonormal system of tangential vectors $\left(\boldsymbol{\tau}_{1}, \ldots, \boldsymbol{\tau}_{d-1}\right)$ on $\Gamma$ and the corresponding tangential divergence $\operatorname{div}_{\tau}$ and gradient $\nabla_{\tau}$. We assume that inside the fractures, the normal direction is a permeability principal direction, such that the permeability tensor decomposes in a tangential part and a normal part as

$$
\Lambda=\Lambda_{f}+\lambda_{f, n} \mathbf{n} \otimes \mathbf{n},
$$

with $\Lambda_{f} \mathbf{n}=0$. On $\Omega_{f}$, we also assume that the permeability and porosity, as well as the constitutive relations for the phase saturations and mobilities do not explicitly depend on the normal component of the spatial coordinate (we will use the index $f$ to denote their projections on $\Gamma$ ). 
To start, we write

$$
\mathbf{q}^{\alpha}=\sum_{i=1}^{d-1}\left(\mathbf{q}^{\alpha} \cdot \boldsymbol{\tau}_{i}\right) \boldsymbol{\tau}_{i}+\left(\mathbf{q}^{\alpha} \cdot \mathbf{n}\right) \mathbf{n}
$$

Let $\mathbf{g}_{\tau}=\mathbf{g}-(\mathbf{g} \cdot \mathbf{n}) \mathbf{n}$. With (2) and (3), (1b) is equivalent to

$$
\begin{aligned}
\sum_{i=1}^{d-1}\left(\mathbf{q}^{\alpha} \cdot \boldsymbol{\tau}_{i}\right) \boldsymbol{\tau}_{i} & =-k_{f}^{\alpha}\left(\mathbf{x}, S_{f}^{\alpha}(\mathbf{x}, p)\right) \Lambda_{f}\left(\nabla_{\tau} u^{\alpha}-\rho^{\alpha} \mathbf{g}_{\tau}\right) \\
\mathbf{q}^{\alpha} \cdot \mathbf{n} & =-k_{f}^{\alpha}\left(\mathbf{x}, S_{f}^{\alpha}(\mathbf{x}, p)\right) \lambda_{f, n}\left(\partial_{\mathbf{n}} u^{\alpha}-\rho^{\alpha} \mathbf{g} \cdot \mathbf{n}\right) .
\end{aligned}
$$

Respectively, the averaged pressure and the integrated tangential Darcy velocity accross the fracture are defined by

$$
u_{f}^{\alpha}=\frac{1}{d_{f}} \int_{-\frac{d_{f}}{2}}^{\frac{d_{f}}{2}} u^{\alpha} \mathrm{d} \mathbf{n} \quad \text { and } \quad \mathbf{q}_{f}^{\alpha}=\sum_{i=1}^{d-1} \int_{-\frac{d_{f}}{2}}^{\frac{d_{f}}{2}}\left(\mathbf{q}^{\alpha} \cdot \boldsymbol{\tau}_{i}\right) \boldsymbol{\tau}_{i} \mathrm{~d} \mathbf{n} .
$$

Furthermore, let us use the approximations

$$
k_{f}^{\alpha}\left(\mathbf{x}, S_{f}^{\alpha}(\mathbf{x}, p)\right) \approx k_{f}^{\alpha}\left(\mathbf{x}, S_{f}^{\alpha}\left(\mathbf{x}, p_{f}\right)\right) \quad \text { and } \quad S_{f}^{\alpha}\left(\mathbf{x}, p_{f}\right) \approx \frac{1}{d_{f}} \int_{-\frac{d_{f}}{2}}^{\frac{d_{f}}{2}} S_{f}^{\alpha}(\mathbf{x}, p) \mathrm{d} \mathbf{n} .
$$

Integration of the conservation equation (1a) and the tangential Darcy law (4a) over the fracture width yields the fracture equations for the hybrid-dimensional model,

$$
\left\{\begin{aligned}
\phi_{f} d_{f} \partial_{t} S_{f}^{\alpha}\left(\mathbf{x}, p_{f}\right)+\operatorname{div}_{\tau}\left(\mathbf{q}_{f}^{\alpha}\right)-\sum_{\mathfrak{a} \in \chi} \gamma_{\mathbf{n}_{\mathfrak{a}}} \mathbf{q}_{m}^{\alpha} & =0 & & \text { on } \Gamma \\
\mathbf{q}_{f}^{\alpha} & =-d_{f} k_{f}^{\alpha}\left(\mathbf{x}, S_{f}^{\alpha}\left(\mathbf{x}, p_{f}\right)\right) \Lambda_{f}\left(\nabla_{\tau} u_{f}-\rho^{\alpha} \mathbf{g}_{\tau}\right) & & \text { on } \Gamma
\end{aligned}\right.
$$

The matrix equations for the hybrid-dimensional model are

$$
\left\{\begin{aligned}
\phi_{m} \partial_{t} S_{m}^{\alpha}\left(\mathbf{x}, p_{m}\right)+\operatorname{div}\left(\mathbf{q}_{m}^{\alpha}\right) & =0 & & \text { on } \Omega \backslash \bar{\Gamma} \\
\mathbf{q}_{m}^{\alpha} & =-k_{m}^{\alpha}\left(\mathbf{x}, S_{m}^{\alpha}\left(\mathbf{x}, p_{m}\right)\right) \Lambda_{m}\left(\nabla u_{m}^{\alpha}-\rho^{\alpha} \mathbf{g}\right) & & \text { on } \Omega \backslash \bar{\Gamma}
\end{aligned}\right.
$$

Moreover, both phases are coupled by the equations

$$
\left(p_{m}, p_{f}\right)=\left(u_{m}^{1}-u_{m}^{2}, u_{f}^{1}-u_{f}^{2}\right), \quad\left(S_{m}^{2}, S_{f}^{2}\right)=1-\left(S_{m}^{1}, S_{f}^{1}\right) .
$$

Hybrid-dimensional $m f$ nonlinear model The matrix and the fracture equations are coupled by Robin boundary conditions imposed at each $\Gamma_{\mathfrak{a}}, \mathfrak{a} \in \chi$. For this, we use a two point approximation of the normal derivative of the pressure in the linear part of (4b), which leads to the definition of the velocity

$$
V_{f, n}^{\alpha, \mathfrak{a}}=\lambda_{f, n}\left(\frac{\gamma_{\mathfrak{a}} u_{m}^{\alpha}-u_{f}^{\alpha}}{d_{f} / 2}-\rho^{\alpha} \gamma_{\mathbf{n}_{\mathfrak{a}}} \mathbf{g}\right) .
$$

In order to transport the saturations between the matrix and the fractures, the mobility has to be upwinded in the definition of the normal flux $\gamma_{\mathbf{n}_{\mathfrak{a}}} \mathbf{q}_{m}^{\alpha}$. For any $a \in \mathbb{R}$, let us set $a^{+}=\max \{0, a\}$ and $a^{-}=-(-a)^{+}$. The resulting equation on $\Gamma_{\mathfrak{a}}, \mathfrak{a} \in \chi$ is

$$
\begin{aligned}
& \gamma_{\mathbf{n}_{\mathfrak{a}}} \mathbf{q}_{m}^{\alpha}=\mathbf{q}_{f, \mathbf{n}_{\mathfrak{a}}}^{\alpha} \\
& \mathbf{q}_{f, \mathbf{n}_{\mathfrak{a}}}^{\alpha}=k_{f}^{\alpha}\left(\mathbf{x}, S_{f}^{\alpha}\left(\mathbf{x}, \gamma_{\mathfrak{a}} p_{m}\right)\right)\left(V_{f, n}^{\alpha, \mathfrak{a}}\right)^{+}+k_{f}^{\alpha}\left(\mathbf{x}, S_{f}^{\alpha}\left(\mathbf{x}, p_{f}\right)\right)\left(V_{f, n}^{\alpha, \mathfrak{a}}\right)^{-} .
\end{aligned}
$$

As illustrated in figure 4, the upwinding of the mobilities in the definition of the flux $\mathbf{q}_{f, \mathbf{n}_{\mathfrak{a}}}^{\alpha}$ takes into account the saturation jump due to discontinuous capillary pressure curves at the matrix-fracture interface.

Model (5),(7) will be referred to as the hybrid-dimensional $m f$ nonlinear model since it accounts for the nonlinear two-phase Darcy flux conservation equations at the $m f$ interfaces. 


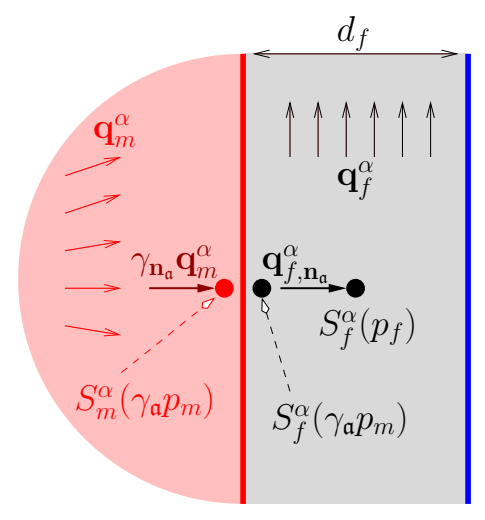

Figure 4: Illustration of the coupling condition $\mathbf{q}_{f, \mathbf{n}_{\mathfrak{a}}}^{\alpha}=\gamma_{\mathbf{n}_{\mathfrak{a}}} \mathbf{q}_{m}^{\alpha}$ for the hybriddimensional $m f$ nonlinear model.

Continuous hybrid-dimensional model In the case of fractures acting as drains for which $\frac{\lambda_{f, n}}{d_{f}} \gg$ $\frac{\left|\Lambda_{m}\right|}{\operatorname{diam}(\Omega)}$, condition (6) is usually further approximated by the pressure continuity condition at the matrix fracture interface $\Gamma$

$$
\gamma_{\mathfrak{a}^{+}(i)} u_{m}^{\alpha}=\gamma_{\mathfrak{a}^{-}(i)} u_{m}^{\alpha}=u_{f},
$$

recovering the condition introduced in [3] for single-phase Darcy flows and in [8, 31, 9] for two-phase flows. The model defined by (5),(8) will be referred to as the continuous hybrid-dimensional model, in the rest of this paper. It will be shown in section 5 that, even in the case of fractures acting as drains, this model can lead to inaccurate results in the case of gravity segregation in the fracture width or highly contrasted capillary pressures at the mf interfaces.

DFN closure conditions Finally, closure conditions are set at the immersed boundary of the fracture network (fracture tips) as well as at the intersection between fractures. For $i \in I$, let $\gamma_{\Sigma_{i}}$ (resp. $\gamma_{\mathbf{n}_{\Sigma_{i}}}$ ) denote the trace (resp. normal trace) operator at the fracture $\Gamma_{i}$ boundary oriented outward to $\Gamma_{i}$, and let $\gamma_{\mathbf{n}_{\partial \Gamma}}$ denote the normal trace operator at the fracture network boundary oriented outward to $\Gamma$. At fracture tips $\partial \Gamma \backslash \partial \Omega$, it is classical to assume homogeneous Neumann boundary conditions in the sense that

$$
\gamma_{\mathbf{n}_{\partial \Gamma}} \mathbf{q}_{f}^{\alpha}=0 \quad \text { on } \partial \Gamma \backslash \partial \Omega, \alpha=1,2,
$$

meaning that the flow at the tip of a fracture can be neglected compared with the flow along the sides of the fracture. At the fracture intersection $\Sigma_{i, j} \backslash \Sigma_{i, 0}, i, j \in I, i \neq j$, we impose the normal flux conservation equations

$$
\sum_{k \in I} \gamma_{\mathbf{n}_{\Sigma_{k}}} \mathbf{q}_{f}^{\alpha}=0 \quad \text { on } \Sigma_{i, j} \backslash \Sigma_{i, 0}, \alpha=1,2,
$$

meaning that the volume at the intersection between fractures is neglected. In addition, we impose continuity of the phase pressures at $\Sigma_{i, j}$

$$
\gamma_{\Sigma_{i}} u_{f}^{\alpha}=\gamma_{\Sigma_{j}} u_{f}^{\alpha} \quad \text { on } \Sigma_{i, j}, \alpha=1,2 .
$$

This amounts to assume a high ratio between the permeability at the intersection and the fracture width compared with the ratio between the tangential permeability of each fracture and its lengh. This assumption typically does not account for a barrier intersecting a drain. In such a case, the pressure continuity could be replaced by a zero flux boundary condition together with discontinuous pressure at intersecting barrier/drain edges. The side(s) on which the zero flux condition is imposed will depend on the modelling assumptions at each intersecting edge. Alternatively, reduced models have been derived in 2D to deal with general fracture intersections [20] but to our knowledge such construction is too complex in $3 \mathrm{D}$ and has not been developed.

Up to now, the only existing, comparable hybrid-dimensional two-phase flow model to the hybriddimensional $m f$ nonlinear model presented above is the model described in $[2,27]$, which is presented 
in global pressure formulation. We adapted here a formulation using the phase pressures as primary unknowns, accounting for complex fracture networks and general invertible capillary pressure functions. As illustrated in the numerical section it can be easily extended to general capillary pressure curves including vanishing capillary pressure in the fractures using a switch of variable formulation as described in [10] (see also [30]). Another difference is, that the hybrid-dimensional $m f$ nonlinear model uses an upwind coupling condition for the matrix-fracture normal fluxes (see (7)). This upwinding is necessary to transport the saturations from the matrix to the fractures. The coupling condition (7) also takes into account gravitational force inside the fractures for the matrix-fracture mass exchange. In the test cases below, we see that this is an important feature for the simulation of gravity dominated flow.

\section{Vertex Approximate Gradient Scheme}

In this section, the VAG scheme, introduced in [14] for diffusive problems on heterogeneous anisotropic media and extended to discrete fractures in [12] for the discontinuous hybrid-dimensional models, is adapted to the hybrid-dimensional two-phase flow models. We consider a finite volume version using lumping both for the accumulation terms and the matrix fracture fluxes. For an adaptation of the VAG scheme to the continuous hybrid-dimensional model, we refer the reader to $[11,9]$.

\subsection{VAG Discretization}

\subsubsection{Polyhedral meshes}

Following [14], we consider generalized polyhedral meshes of $\Omega$. Let $\mathcal{M}$ be the set of cells that are disjoint open polyhedral subsets of $\Omega$ such that $\bigcup_{K \in \mathcal{M}} \bar{K}=\bar{\Omega}$. For all $K \in \mathcal{M}, \mathbf{x}_{K}$ denotes the so-called "center" of the cell $K$ under the assumption that $K$ is star-shaped with respect to $\mathbf{x}_{K}$. Let $\mathcal{F}$ denote the set of (not necessarily planar) faces of the mesh. We denote by $\mathcal{V}$ the set of vertices of the mesh. Let $\mathcal{F}_{K}, \mathcal{V}_{\sigma}$ respectively denote the set of faces of $K$ and vertices of $\sigma \in \mathcal{F}$. The set of edges of the mesh is denoted by $\mathcal{E}$ and $\mathcal{E}_{\sigma}$ denotes the set of edges of the face $\sigma \in \mathcal{F}$. Let $\mathcal{M}_{\sigma}$ denote the set of cells sharing the face $\sigma \in \mathcal{F}$. We denote by $\mathcal{F}_{\text {ext }}$ the subset of faces $\sigma \in \mathcal{F}$ such that $\mathcal{M}_{\sigma}$ has only one element, and we set $\mathcal{V}_{\text {ext }}=\bigcup_{\sigma \in \mathcal{F}_{\text {ext }}} \mathcal{V}_{\sigma}$. The mesh is assumed to be conforming in the sense that for all $\sigma \in \mathcal{F} \backslash \mathcal{F}_{\text {ext }}$, the set $\mathcal{M}_{\sigma}$ contains exactly two cells. It is assumed that for each face $\sigma \in \mathcal{F}$, there exists a so-called "center" of the face $\mathbf{x}_{\sigma}$ such that

$$
\mathbf{x}_{\sigma}=\sum_{s \in \mathcal{V}_{\sigma}} \beta_{\sigma, s} \mathbf{x}_{s}, \text { with } \sum_{s \in \mathcal{V}_{\sigma}} \beta_{\sigma, s}=1,
$$

where $\beta_{\sigma, s} \geq 0$ for all $s \in \mathcal{V}_{\sigma}$. The face $\sigma$ is assumed to match with the union of the triangles $T_{\sigma, e}$ defined by the face center $\mathbf{x}_{\sigma}$ and each of its edges $e \in \mathcal{E}_{\sigma}$. The mesh is assumed to be conforming w.r.t. the fracture network $\Gamma$ in the sense that there exist subsets $\mathcal{F}_{\Gamma_{i}}, i \in I$ of $\mathcal{F}$ such that

$$
\bar{\Gamma}_{i}=\bigcup_{\sigma \in \mathcal{F}_{\Gamma_{i}}} \bar{\sigma} .
$$

We will denote by $\mathcal{F}_{\Gamma}$ the set of fracture faces $\bigcup_{i \in I} \mathcal{F}_{\Gamma_{i}}$. Similarly, we will denote by $\mathcal{V}_{\Gamma}$ the set of fracture vertices $\bigcup_{\sigma \in \mathcal{F}_{\Gamma}} \mathcal{V}_{\sigma}$. We also define a submesh $\mathcal{T}$ of tetrahedra, where each tetrahedron $D_{K, \sigma, e}$ is the convex hull of the cell center $\mathbf{x}_{K}$ of $K$, the face center $\mathbf{x}_{\sigma}$ of $\sigma \in \mathcal{F}_{K}$ and the edge $e \in \mathcal{E}_{\sigma}$. Similarly we define a triangulation $\Delta$ of $\Gamma$, such that we have:

$$
\mathcal{T}=\bigcup_{K \in \mathcal{M}, \sigma \in \mathcal{F}_{K}, e \in \mathcal{E}_{\sigma}}\left\{D_{K, \sigma, e}\right\} \quad \text { and } \quad \Delta=\bigcup_{\sigma \in \mathcal{F}_{\Gamma}, e \in \mathcal{E}_{\sigma}}\left\{T_{\sigma, e}\right\} .
$$

The mesh is also assumed to be conforming w.r.t. the rock types, in the sense that we have a well defined rock type for each cell and fracture face, denoted by $r t_{K}$ and $r t_{\sigma}$, for $K \in \mathcal{M}$ and $\sigma \in \mathcal{F}_{\Gamma}$. 


\subsubsection{Degrees of freedom}

The set of matrix and fracture degrees of freedom is denoted by $d o f_{\mathcal{D}}=d o f_{\mathcal{D}_{m}} \cup d o f_{\mathcal{D}_{f}}$, with $\operatorname{dof}_{\mathcal{D}_{m}} \cap$ $d o f_{\mathcal{D}_{f}}=\emptyset$. The real vector space of discrete unknowns is denoted by $X_{\mathcal{D}}=\mathbb{R} \# \operatorname{dof}_{\mathcal{D}}$. For $u_{\mathcal{D}} \in X_{\mathcal{D}}$ and $\nu \in d o f_{\mathcal{D}}$, we denote by $u_{\nu}$ the $\nu$ th component of $u_{\mathcal{D}}$. To account for Dirichlet boundary conditions on $\partial \Omega$ and $\Sigma_{0}$ we introduce the subsets $\operatorname{dof}_{D i r_{m}} \subset d o f_{\mathcal{D}_{m}}$, and $\operatorname{dof}_{D_{i r_{f}}} \subset \operatorname{dof}_{\mathcal{D}_{f}}$, and we set $d o f_{\text {Dir }}=d o f_{\text {Dir }_{m}} \cup d o f_{D_{\text {Dirf }}}$, and $X_{\mathcal{D}}^{0}=\left\{u_{\mathcal{D}} \in X_{\mathcal{D}} \mid u_{\nu}=0\right.$ for all $\left.\nu \in d o f_{\text {Dir }}\right\}$. Concretely, we consider the set of d.o.f. as illustrated in figure 5 .
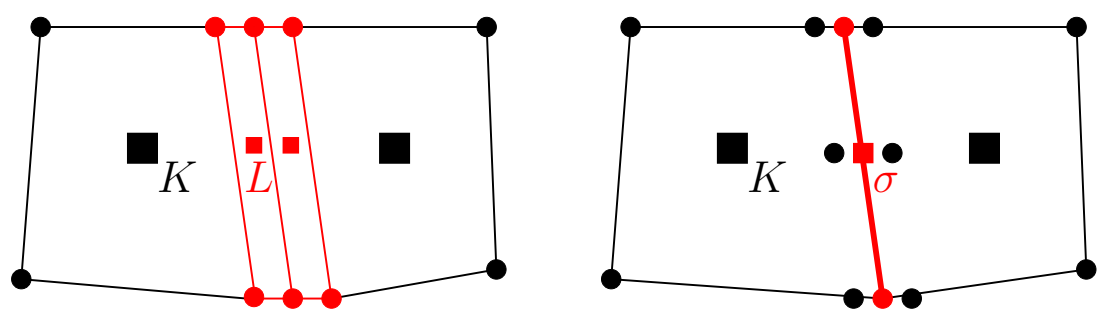

Figure 5: Illustration of d.o.f. in 2D for a matrix domain (in black) intersected by a fracture (in red) for the equi-dimensional (left) and discontinuous hybrid-dimensional (right) models.

Equi-dimensional model Since the equi-dimensional approach does not reduce the fracture dimension, we have to deal with small fracture cells. The standard VAG scheme, as introduced in [14] for single-phase flow and in $[16,17]$ for two-phase flows, which is based on cell and node unknowns, is used for this model.

Discontinuous hybrid-dimensional models The set $d o f_{\mathcal{D}_{f}}$ of fracture d.o.f. relate to face unknowns and node unknowns. This corresponds to the d.o.f. of the standard VAG scheme in dimension $d-1$ on the fracture network. Let us split $\operatorname{dof}_{\mathcal{D}_{m}}$ in the interfacial d.o.f. $\quad \operatorname{dof}_{\mathcal{D}_{m}}^{\Gamma}$, located at the fracture network, and the bulk d.o.f. $\operatorname{dof}_{\mathcal{D}_{m}} \backslash \operatorname{dof}_{\mathcal{D}_{m}}^{\Gamma}$. The unknowns at the interfaces are necessary in order to capture the pressure discontinuities between the matrix and fractures. Since we have to take into account these pressure jumps for all fracture unknowns and all $m f$ interfaces, $d o f_{\mathcal{D}_{m}}^{\Gamma}$ refers to node unknowns plus face unknowns, which are related to the fracture d.o.f. one-to-one for each side of the DFN. Most often, the fractures cut the matrix domain in two. For immersed fracture tips or multiple fracture intersections, however, we have up to as many unknowns as fractures connected to the spot (cf. figure 6). The set $\operatorname{dof}_{\mathcal{D}_{m}} \backslash d o f_{\mathcal{D}_{m}}^{\Gamma}$ corresponds to cell unknowns and node unknowns, which are the classical unknowns for the VAG scheme in $d$ dimensions. We refer to [12], for a more detailed presentation.
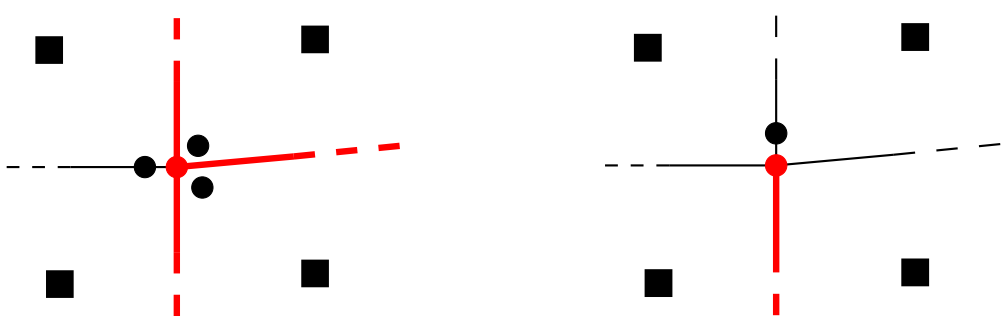

Figure 6: Zoom on the fracture intersection for three fractures (left) and on the fracture tip for one immersed fracture (right). Illustrated are four cells (indicated as boxes) and the d.o.f. at the singular point for the discontinuous hybrid-dimensional models (interfacial d.o.f. in black and fracture d.o.f. in red). 


\subsection{3 $\mathbb{P}_{1}$ Finite Element basis functions}

The basis functions we use to discretize the diffusion terms, denoted by $\eta_{\nu}, \nu \in d o f_{\mathcal{D}}$, in the following, are the $\mathbb{P}_{1}$ Finite Element basis functions on the tetrahedral submesh $\mathcal{T}$, for $\nu \in \operatorname{dof}_{\mathcal{D}_{m}}$ and on the triangulation $\Delta$ of the DFN, for $\nu \in d o f_{\mathcal{D}_{f}}$. The discrete gradients are then defined as the $\mathbb{P}_{1}$ Finite Element gradients on each of the connected matrix subdomains and on the DFN, respectively. For the treatment of the jump terms at the matrix-fracture interfaces, the basis functions are lumped onto a dual mesh of the triangulation $\Delta$. For a more detailed presentation of the construction of the basis functions and discrete gradients, we refer to [12]. To respect the heterogeneities of the media, the $V A G$ reconstructions of the accumulation terms are piecewise constant, as described below.
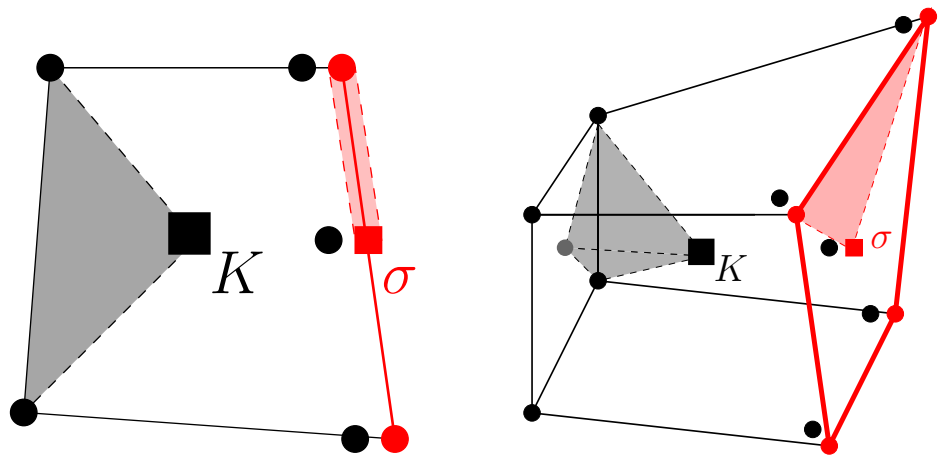

Figure 7: 2D (left) resp. 3D (right) cell $K$ touching a fracture face $\sigma$. Illustration of the simplices on which the matrix (grey) and fracture (red) discrete gradients are constant. The facial unknown located at the coordinate in light grey is eliminated by barycentric interpolation.

\subsubsection{Control volumes}

As mentioned above, the mesh is conforming with the partition in rock types. So, we introduce, for any given $K \in \mathcal{M}, \quad$ a partition of $K$, which takes the general form $\left\{\omega_{K}^{\nu}\right\}_{\nu \in\{K\} \cup\left(\operatorname{dof}_{K} \cap \operatorname{dof}_{\mathcal{D}_{m}}\right) \backslash \text { dof }_{\text {Dir }}}$ and for any given $\sigma \in \mathcal{F}_{\Gamma}$ a partition $\left\{\omega_{\sigma}^{\nu}\right\}_{\nu \in\{\sigma\} \cup\left(\text { dof }_{\sigma} \cap \text { dof }_{\mathcal{D}_{f}}\right) \backslash \text { dof }_{\text {Dir }}}$ of $\sigma$. Here, $d o f_{K}$ and $d o f_{\sigma}$ stand for the d.o.f. connected to $K$ and $\sigma$, respectively, as explained below. The VAG reconstructions of the accumulation terms are piecewise constant on these distributions, and therefore respect the heterogeneities of the media (see [13]). Integrating the reconstructed accumulations shows that, in the numerical scheme, we do not need to provide these partitions explicitly, but only have to define their corresponding volumes.

\subsection{Finite Volume Formulation of the hybrid-dimensional $m f$ nonlinear model}

For $\nu \in d o f_{\mathcal{D}}$, let us denote by $d o f_{\nu} \subset d o f_{\mathcal{D}}$ the set consisting of all $\nu^{\prime}$, such that there is a discrete flux connection between $\nu$ and $\nu^{\prime}$ as illustrated in figure 8. More concretely, for the hybrid-dimensional $m f$ nonlinear model, these sets are defined as follows. The denomination of the different types of d.o.f. (i.e. $\left.K, \sigma, \nu_{m}, \nu_{m}^{\prime}, \nu_{f}\right)$ here is consistent with the notations in figure 8 .

- For $K \in \mathcal{M}: \operatorname{dof} f_{K}=\left\{\nu_{m} \in \operatorname{dof}_{\mathcal{D}_{m}} \backslash d o f_{\mathcal{D}_{m}}^{\Gamma} \mid \mathbf{x}_{\nu_{m}} \in \partial K\right\} \cup\left\{\nu_{m} \in \operatorname{dof}_{\mathcal{D}_{m}}^{\Gamma} \mid \mathbf{x}_{\nu_{m}} \in \partial K\right.$ and $\nu_{m}$ refers to an interfacial unknown located on the same side of $\Gamma$ as $K\}$

- For $\sigma \in \mathcal{F}_{\Gamma}: d o f_{\sigma}=\left\{\nu_{f} \in d o f_{\mathcal{D}_{f}} \mid \mathbf{x}_{\nu_{f}} \in \partial \sigma\right\} \cup\left\{\nu_{m} \in d o f_{\mathcal{D}_{m}}^{\Gamma} \mid \mathbf{x}_{\nu_{m}}=\mathbf{x}_{\sigma}\right\}$

- For $\nu_{m} \in d o f_{\mathcal{D}_{m}}^{\Gamma}: d o f_{\nu_{m}}=\mathcal{M}_{\nu_{m}} \cup\left\{\nu_{f} \in \operatorname{dof}_{\mathcal{D}_{f}} \mid \mathbf{x}_{\nu_{f}}=\mathbf{x}_{\nu_{m}}\right\}$

Since the fluxes connect exactly two unknowns, the definition of the sets $d o f_{\nu}$ is complemented by

$$
\nu^{\prime} \in d o f_{\nu} \Longleftrightarrow \nu \in d o f_{\nu^{\prime}} .
$$

The well defined constitutive relations for the mobilities and saturations for each of these rock types are then denoted by $k^{\alpha}\left(r t_{\nu}, \cdot\right)$ and $S^{\alpha}\left(r t_{\nu}, \cdot\right), \nu \in \mathcal{M} \cup \mathcal{F}_{\Gamma}$, respectively. In order to calculate the fracture mobility for the nonlinear matrix-fracture two point fluxes of the discontinuous hybriddimensional models, for nodal unknowns, we have to define fracture node rock types, in addition to 
cell and fracture face rock types. The fracture node rock types serve exclusively for this purpose and its introduction is consistent with our pressure continuity assumption at fracture intersections which assumes that the properties of intersecting fractures are not too contrasted.

For any $\nu \in d o f_{K}$ the discrete matrix-matrix-fluxes are defined as $-F_{\nu K}^{\alpha}\left(u_{\mathcal{D}}^{1}, u_{\mathcal{D}}^{2}\right)=F_{K \nu}^{\alpha}\left(u_{\mathcal{D}}^{1}, u_{\mathcal{D}}^{2}\right)=k^{\alpha}\left(r t_{K}, S^{\alpha}\left(r t_{K}, p_{K}\right)\right) \cdot f_{K \nu}^{\alpha}\left(u_{\mathcal{D}}^{\alpha}\right)^{+}+k^{\alpha}\left(r t_{K}, S^{\alpha}\left(r t_{K}, p_{\nu}\right)\right) \cdot f_{K \nu}^{\alpha}\left(u_{\mathcal{D}}^{\alpha}\right)^{-}$,

where

$$
-f_{\nu K}^{\alpha}\left(u_{\mathcal{D}}^{\alpha}\right)=f_{K \nu}^{\alpha}\left(u_{\mathcal{D}}^{\alpha}\right)=\sum_{\nu^{\prime} \in d o f_{K}} T_{K}^{\nu \nu^{\prime}}\left(u_{K}^{\alpha}-u_{\nu^{\prime}}^{\alpha}-\rho^{\alpha}\left(\mathbf{x}_{K}-\mathbf{x}_{\nu^{\prime}}\right) \cdot \mathbf{g}\right)
$$

with transmissivities

$$
T_{K}^{\nu \nu^{\prime}}=\int_{K} \Lambda_{m} \nabla \eta_{\nu} \nabla \eta_{\nu^{\prime}} \mathrm{d} \mathbf{x}
$$

For all $\nu \in \operatorname{dof}_{\sigma} \backslash d o f_{\mathcal{D}_{m}}^{\Gamma}$ the discrete fracture-fracture-fluxes are defined as

$$
-F_{\nu \sigma}^{\alpha}\left(u_{\mathcal{D}}^{1}, u_{\mathcal{D}}^{2}\right)=F_{\sigma \nu}^{\alpha}\left(u_{\mathcal{D}}^{1}, u_{\mathcal{D}}^{2}\right)=k^{\alpha}\left(r t_{\sigma}, S^{\alpha}\left(r t_{\sigma}, p_{\sigma}\right)\right) \cdot f_{\sigma \nu}^{\alpha}\left(u_{\mathcal{D}}^{\alpha}\right)^{+}+k^{\alpha}\left(r t_{\sigma}, S^{\alpha}\left(r t_{\sigma}, p_{\nu}\right)\right) \cdot f_{\sigma \nu}^{\alpha}\left(u_{\mathcal{D}}^{\alpha}\right)^{-},
$$

where

$$
-f_{\nu \sigma}^{\alpha}\left(u_{\mathcal{D}}^{\alpha}\right)=f_{\sigma \nu}^{\alpha}\left(u_{\mathcal{D}}^{\alpha}\right)=\sum_{\nu^{\prime} \in d o f_{\sigma}} T_{\sigma}^{\nu \nu^{\prime}}\left(u_{\sigma}^{\alpha}-u_{\nu^{\prime}}^{\alpha}-\rho^{\alpha}\left(\mathbf{x}_{\sigma}-\mathbf{x}_{\nu^{\prime}}\right) \cdot \mathbf{g}\right),
$$

with transmissivities

$$
T_{\sigma}^{\nu \nu^{\prime}}=\int_{\sigma} d_{f} \Lambda_{f} \nabla_{\tau} \eta_{\nu} \nabla_{\tau} \eta_{\nu^{\prime}} \mathrm{d} \tau(\mathbf{x})
$$

Let us further introduce the set of matrix-fracture $(m f)$ connectivities

$$
\mathcal{C}=\left\{\left(\nu_{m}, \nu_{f}\right) \mid \nu_{m} \in \operatorname{dof}_{\mathcal{D}_{m}}^{\Gamma}, \nu_{f} \in \operatorname{dof} f_{\mathcal{D}_{f}} \text { s.t. } \mathbf{x}_{\nu_{m}}=\mathbf{x}_{\nu_{f}}\right\} .
$$

The $m f$-fluxes are defined as

$$
\begin{aligned}
-F_{\nu_{f} \nu_{m}}^{\alpha}\left(u_{\mathcal{D}}^{1}, u_{\mathcal{D}}^{2}\right)=F_{\nu_{m} \nu_{f}}^{\alpha}\left(u_{\mathcal{D}}^{1}, u_{\mathcal{D}}^{2}\right)= & k^{\alpha}\left(r t_{\nu_{f}}, S^{\alpha}\left(r t_{\nu_{f}}, p_{\nu_{m}}\right)\right) \cdot f_{\nu_{m} \nu_{f}}^{\alpha}\left(u_{\mathcal{D}}^{\alpha}\right)^{+} \\
& +k^{\alpha}\left(r t_{\nu_{f}}, S^{\alpha}\left(r t_{\nu_{f}}, p_{\nu_{f}}\right)\right) \cdot f_{\nu_{m} \nu_{f}}^{\alpha}\left(u_{\mathcal{D}}^{\alpha}\right)^{-},
\end{aligned}
$$

where

$$
-f_{\nu_{f} \nu_{m}}^{\alpha}\left(u_{\mathcal{D}}^{\alpha}\right)=f_{\nu_{m} \nu_{f}}^{\alpha}\left(u_{\mathcal{D}}^{\alpha}\right)=T_{\nu_{m} \nu_{f}}\left(u_{\nu_{m}}^{\alpha}-u_{\nu_{f}}^{\alpha}-\frac{\rho^{\alpha} d_{f}}{2} \gamma_{\mathbf{n}_{\mathfrak{a}}} \mathbf{g}\right)
$$

with transmissivities

$$
T_{\nu_{m} \nu_{f}}=\sum_{\substack{T \in \Delta \\ \text { s.t. } \mathbf{x}_{\nu_{f}} \in \bar{T}}} \frac{1}{3} \int_{T} \frac{2 \lambda_{f, n}}{d_{f}} \mathrm{~d} \tau(\mathbf{x}) .
$$

We observe that for the VAG scheme, the fluxes $F_{\nu_{m} \nu_{f}}^{\alpha}$ are two point flux approximations.

Let $0=t^{0}<t^{1}<\cdots<t^{N}=T$, with $\Delta t^{n}=t^{n}-t^{n-1}$ be a time discretization. Given $p_{\mathcal{D}}^{0} \in X_{\mathcal{D}}$, and using an implicit Euler time integration, the Finite Volume formulation of (5),(7) reads as follows: Find $\left\{u_{\mathcal{D}}^{\alpha}\right\}_{\alpha=1,2} \in\left(X_{\mathcal{D}}^{0}\right)^{2 N}$ such that for all $n \in\{1, \ldots, N\}$ 

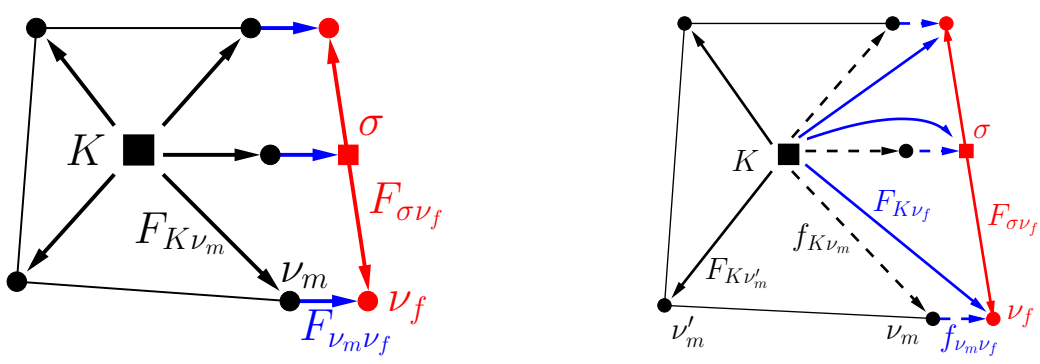

Figure 8: VAG $m m$-fluxes (black), $m f$-fluxes (blue) and $f f$-fluxes (red) on a $2 \mathrm{D}$ cell touching a fracture. Hybrid-dimensional $m f$ nonlinear model (left) and $m f$ linear model (right). The fluxes indicated by solid lines and capital $F$ correspond to nonlinear fluxes incorporating the mobility and appear in the flux conservation equations of each of the two control volumes they connect. The dashed fluxes $f$ for the $m f$ linear model, are linearly depending on the pressure. Consequently, the equations for interfacial $\nu_{m} \in \operatorname{dof}_{\mathcal{D}_{m}}^{\Gamma}$ are the linear flux conservation equations (14), for this model.
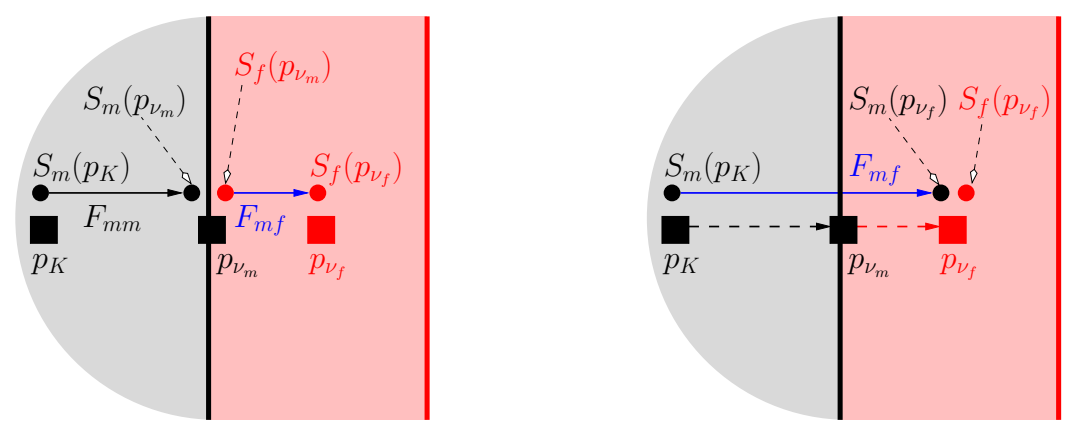

Figure 9: Sketch of how VAG accounts for saturation jumps at the $m f$ interfaces. The matrix and interfacial unknowns are indicated by the black boxes and the fracture unknown by the red box. For the hybrid-dimensional $m f$ nonlinear model (left figure), the well defined capillary pressure $p_{\nu_{m}}$ for the interfacial unknown is used to calculate the matrix and fracture saturations at the interface, $S_{m}\left(p_{\nu_{m}}\right)$ and $S_{f}\left(p_{\nu_{m}}\right)$, respectively, as the inverse of the matrix and fracture capillary pressure curves. $F_{m m}$ is then calculated with $S_{m}\left(p_{\nu_{m}}\right)$ and $F_{m f}$ is calculated with $S_{f}\left(p_{\nu_{m}}\right)$. For the hybrid-dimensional $m f$ linear models, monophasic (linear) flux conservation is used to determine the interfacial pressures (dotted arrays). To evaluate the fracture upwind mobility for the nonlinear fluxes between matrix cell and fracture unknowns, the fracture capillary pressure is used to calculate either $S_{m}\left(p_{\nu_{f}}\right)$, for the $m$ upwind scheme, which hence accounts for the saturation jumps at the interface, or $S_{f}\left(p_{\nu_{f}}\right)$, for the $f$ upwind scheme, using the fracture rock type saturation function.

for all $K \in \mathcal{M}$ :

$$
\left|\omega_{K}^{K}\right| \phi_{K} \frac{S^{\alpha}\left(r t_{K}, p_{K}^{n}\right)-S^{\alpha}\left(r t_{K}, p_{K}^{n-1}\right)}{\Delta t^{n}}+\sum_{\nu \in d o f_{K}} F_{K \nu}^{\alpha}\left(u_{\mathcal{D}}^{1, n}, u_{\mathcal{D}}^{2, n}\right)=0
$$

for all $\nu_{m} \in \operatorname{dof}_{\mathcal{D}_{m}} \backslash\left(\mathcal{M} \cup \operatorname{dof}{ }_{D i r_{m}}\right)$ :

$$
\sum_{K \in \mathcal{M}_{\nu_{m}}}\left|\omega_{K}^{\nu_{m}}\right| \phi_{K} \frac{S^{\alpha}\left(r t_{K}, p_{\nu_{m}}^{n}\right)-S^{\alpha}\left(r t_{K}, p_{\nu_{m}}^{n-1}\right)}{\Delta t^{n}}+\sum_{\nu \in \operatorname{dof}_{\nu_{m}}} F_{\nu_{m} \nu}^{\alpha}\left(u_{\mathcal{D}}^{1, n}, u_{\mathcal{D}}^{2, n}\right)=0
$$

for all $\sigma \in \mathcal{F}_{\Gamma}$ :

for all $\nu_{f} \in \operatorname{dof}_{\mathcal{D}_{f}} \backslash\left(\mathcal{F}_{\Gamma} \cup \operatorname{dof} \operatorname{Dir}_{f}\right)$ :

$$
\left|\omega_{\sigma}^{\sigma}\right| \phi_{\sigma} \frac{S^{\alpha}\left(r t_{\sigma}, p_{\sigma}^{n}\right)-S^{\alpha}\left(r t_{\sigma}, p_{\sigma}^{n-1}\right)}{\Delta t^{n}}+\sum_{\nu \in d o f_{\sigma}} F_{\sigma \nu}^{\alpha}\left(u_{\mathcal{D}}^{1, n}, u_{\mathcal{D}}^{2, n}\right)=0
$$

$$
\sum_{\sigma \in \mathcal{F}_{\Gamma, \nu_{f}}}\left|\omega_{\sigma}^{\nu_{f}}\right| \phi_{\sigma} \frac{S^{\alpha}\left(r t_{\sigma}, p_{\nu_{f}}^{n}\right)-S^{\alpha}\left(r t_{\sigma}, p_{\nu_{f}}^{n-1}\right)}{\Delta t^{n}}+\sum_{\nu \in \operatorname{dof}_{\nu_{f}}} F_{\nu_{f} \nu}^{\alpha}\left(u_{\mathcal{D}}^{1, n}, u_{\mathcal{D}}^{2, n}\right)=0 .
$$


In (12), the set $\mathcal{M}_{\nu_{m}}$ stands for the set of indices $\left\{K \in \mathcal{M} \mid \nu_{m} \in d o f_{K}\right\}$, and $\mathcal{F}_{\Gamma, \nu_{f}}$ stands for the set $\left\{\sigma \in \mathcal{F}_{\Gamma} \mid \nu_{f} \in \operatorname{dof}_{\sigma}\right\}$.

\subsection{Hybrid-dimensional $m f$ linear models}

For practical considerations, the equations for the interfacial unknowns $\nu_{m} \in \operatorname{dof}_{\mathcal{D}_{m}}^{\Gamma}$ are quite costly to solve, since the interfacial accumulation volumes $\bigcup_{K \in \mathcal{M}_{\nu_{m}}} \omega_{K, \nu_{m}}$ generally have to be chosen significantly smaller than the accumulation volumes at unknowns located inside the fractures. In this section, we propose a model that uses linear equations for $\nu_{m} \in d o f_{\mathcal{D}_{m}}^{\Gamma}$ to calculate the phase pressures at the matrix-fracture interfaces. On the other hand, interfacial saturations are not calculated, but matrix-fracture mass transfer uses the saturations inside the fractures, instead.

As illustrated in figure 8 for the hybrid-dimensional $m f$-linear model, the nonlinear flux connections defined by the sets $d o f_{\nu}$ are modified for $\nu \in d o f_{\mathcal{D}} \backslash d o f_{\mathcal{D}_{m}}^{\Gamma}$ as follows:

- For $K \in \mathcal{M}: \operatorname{dof}_{K}=\left\{\nu \in \operatorname{dof}_{\mathcal{D}} \backslash d o f_{\mathcal{D}_{m}}^{\Gamma} \mid \mathbf{x}_{\nu} \in \partial K\right\}$

- For $\sigma \in \mathcal{F}_{\Gamma}: d o f_{\sigma}=\left\{\nu_{f} \in d o f_{\mathcal{D}_{f}} \mid \mathbf{x}_{\nu_{f}} \in \partial \sigma\right\} \cup \mathcal{M}_{\sigma}$

together with the relation (9) for $\nu \in \operatorname{dof}_{\mathcal{D}} \backslash d o f_{\mathcal{D}_{m}}^{\Gamma}$.

Let us denote by $\nu_{f}\left(\nu_{m}\right) \in d o f_{\mathcal{D}_{f}}$ for each $\nu_{m} \in d o f_{\mathcal{D}_{m}}^{\Gamma}$, the unique d.o.f. $\nu_{f} \in d o f_{\mathcal{D}_{f}}$ such that $\mathbf{x}_{\nu_{m}}=\mathbf{x}_{\nu_{f}}$. Unlike for the hybrid-dimensional $m f$ nonlinear model, the d.o.f. $\nu_{m} \in \operatorname{dof}_{\mathcal{D}_{m}}^{\Gamma}$ are not linked to their neighbours via nonlinear fluxes. Also, in the above definition (see also figure 8), for $K \in \mathcal{M}_{\nu_{m}}, \nu_{m}$ is replaced by $\nu_{f}\left(\nu_{m}\right)$ in $d o f_{K}$ (and $K$ is added to $d o f_{\nu_{f}\left(\nu_{m}\right)}$ ). The mass exchange between matrix and DFN is accounted for by the nonlinear fluxes

$$
F_{K \nu_{f}}^{\alpha}\left(u_{\mathcal{D}}^{1}, u_{\mathcal{D}}^{2}\right)=k^{\alpha}\left(r t_{K}, S^{\alpha}\left(r t_{K}, p_{K}\right)\right) \cdot f_{K \nu_{m}}^{\alpha}\left(u_{\mathcal{D}}^{\alpha}\right)^{+}+k^{\alpha}\left(r t_{K}, S^{\alpha}\left(r t_{K}, p_{\nu_{f}}\right)\right) \cdot f_{K \nu_{m}}^{\alpha}\left(u_{\mathcal{D}}^{\alpha}\right)^{-},
$$

with $\nu_{f}=\nu_{f}\left(\nu_{m}\right)$ and $K \in \mathcal{M}_{\nu_{m}}$. For the $m f$-linearized model, the interfacial control volumes are set to zero, i.e. $\omega_{\nu_{m}}=\emptyset$ for $\nu_{m} \in d o f_{\mathcal{D}_{m}}^{\Gamma}$. Equation (12) becomes (for all $n \in\{1, \ldots, N\}$ )

$$
\sum_{K \in \mathcal{M}_{\nu_{m}}} f_{K \nu_{m}}^{\alpha}\left(u_{\mathcal{D}}^{\alpha, n}\right)=f_{\nu_{m} \nu_{f}}^{\alpha}\left(u_{\mathcal{D}}^{\alpha, n}\right) \quad \text { for all } \nu_{m} \in \operatorname{dof}_{\mathcal{D}_{m}}^{\Gamma}
$$

again with $\nu_{f}=\nu_{f}\left(\nu_{m}\right)$. We see that the nonlinear equations have been replaced by linear equations for the interfacial unknowns, which constitutes the main advantage of this model compared with the hybrid-dimensional $m f$ nonlinar model. This is equivalent to the procedure of eliminating interfacial unknowns by harmonic averaging of the half transmissibilities for cell centered schemes, as described in [25], for example. This model will be referred to as the hybrid-dimensional $m f$ linear $m$ upwind model, in the following.

The choice (13) of the $m f$ fluxes captures the jump of the saturation at the $m f$ interfaces and hence can typically accounts accurately for a matrix acting as a barrier for the non wetting phase. On the other hand, it does not provide a good approximation of the capillary pressure inside the fracture when the fracture is filled with the non wetting phase. This is the reason why the following second choice of the $m f$ fluxes using the fracture rocktype will also be tested

$$
F_{K \nu_{f}}^{\alpha}\left(u_{\mathcal{D}}^{1}, u_{\mathcal{D}}^{2}\right)=k^{\alpha}\left(r t_{K}, S^{\alpha}\left(r t_{K}, p_{K}\right)\right) \cdot f_{K \nu_{m}}^{\alpha}\left(u_{\mathcal{D}}^{\alpha}\right)^{+}+k^{\alpha}\left(r t_{\nu_{f}}, S^{\alpha}\left(r t_{\nu_{f}}, p_{\nu_{f}}\right)\right) \cdot f_{K \nu_{m}}^{\alpha}\left(u_{\mathcal{D}}^{\alpha}\right)^{-} .
$$

This second choice does not capture exactly the jump of the saturation at the $m f$ interfaces but provides a better approximation of the capillary pressure inside the fracture when the fracture is filled with the non wetting phase. We will refer to this model as the hybrid-dimensional $m f$ linear $f$ upwind model, in the following.

Note that the continuous hybrid-dimensional model $[9,10]$ (see also $[8,31,30]$ ) corresponds to the $m$ upwind choice (13) and is recovered by replacing equation (14) by the phase pressure continuity equation $u_{\nu_{m}}^{\alpha}=u_{\nu_{f}}^{\alpha}$. In practice, the continuous hybrid-dimensional model $[9,10]$ provides very similar results than hybrid-dimensional $m f$ linear $m$ upwind model in the case of fractures acting as drains but (14) also accounts for fractures acting as barriers. 


\subsection{Outlook}

In section 5, we show numerical tests and compare the three discontinuous hybrid-dimensional models w.r.t. the equi-dimensional model. The continuous hybrid-dimensional model $[9,10]$ is not included in the tests since it provides basically the same results than the hybrid-dimensional $m f$ linear $m$ upwind model in the case of fractures acting as drains. As a short outlook, we already state here the most striking results.

\begin{tabular}{|c|c|c|c|c|c|c|}
\hline & & equi-dim. & $\begin{array}{c}\text { cont. } \\
\text { hybrid dim. }\end{array}$ & $\begin{array}{l}\text { hybrid dim. } \\
m f \text { nonlinear }\end{array}$ & $\begin{array}{c}\text { hybrid dim. } \\
m f \text { linear } m \text { upwind }\end{array}$ & $\begin{array}{l}\text { hybrid dim. } \\
m f \text { linear } f \text { upwind }\end{array}$ \\
\hline \multicolumn{2}{|c|}{ gravity inside DFN (normal dir.) } & $\checkmark$ & $\mathrm{X}$ & $\checkmark$ & $\mathrm{X}$ & $\mathrm{X}$ \\
\hline \multicolumn{2}{|c|}{ fracture normal permeability } & $\checkmark$ & $\mathrm{X}$ & $\checkmark$ & $\checkmark$ & $\checkmark$ \\
\hline \multirow{2}{*}{$\begin{array}{l}\text { accuracy of solu- } \\
\text { tion for discontinuous } \\
\text { capillary pressure }\end{array}$} & $\begin{array}{l}\text { capture the saturation } \\
\text { jumps at mf interfaces }\end{array}$ & reference & $\checkmark$ & $\checkmark$ & $\checkmark$ & $x$ \\
\hline & $\begin{array}{l}\text { good approximation of the } \\
\text { capillary pressure inside the fractures }\end{array}$ & reference & $x$ & $\checkmark$ & $x$ & $\checkmark$ \\
\hline
\end{tabular}

Table 1: Performance of different types of DFM models

\section{Tracer test case}

The models in this section describe the transport of a tracer in a solvant through fractured porous media. It can be seen as a special case of the two-phase flow models presented above assuming that the phase mobilities satisfy $k^{\alpha}\left(S^{\alpha}\right)=S^{\alpha}$, that the capillarity vanishes and that the gravity field is set to zero. In the following, the primary unknowns are denoted by $u$ for the pressure and $c$ for the tracer concentration.
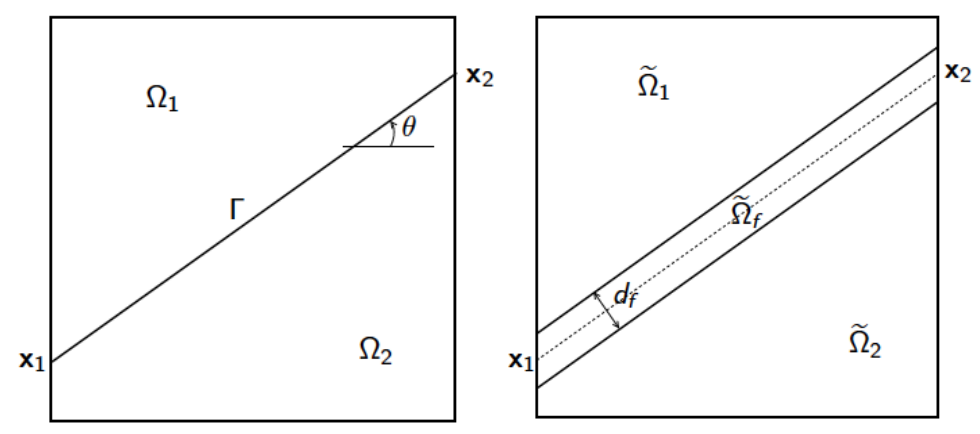

Figure 10: Matrix and fracture domains for the hybrid-dimensional (left) and equi-dimensional (right) tracer test case.

\subsection{Analytical solution for the hybrid-dimensional model}

Let us denote by $(x, y)$ the Cartesian coordinates of $\mathbf{x}$ and let us set $\Omega=(0,1)^{2}, \mathbf{x}_{1}=\left(0, \frac{1}{4}\right), \theta \in$ $\left(0, \arctan \left(\frac{3}{4}\right)\right), \mathbf{x}_{2}=\left(1, \frac{1}{4}+\tan (\theta)\right)$. Let $\Omega_{1}=\left\{(x, y) \in \Omega \mid y>\frac{1}{4}+x \tan (\theta)\right\}$, and $\Omega_{2}=\Omega \backslash \frac{4}{\Omega_{1}}$. As exhibited in the left Figure 10, we consider a single fracture defined by $\Gamma=\left(\mathbf{x}_{1}, \mathbf{x}_{2}\right)=\partial \Omega_{1} \cap \partial \Omega_{2}$ with tangential permeability $\lambda_{f}>0$, normal permeability $\lambda_{f, n}$ and width $d_{f}>0$. The matrix permeability is isotropic and set to $\lambda_{m}=1$. The matrix and fracture porosities are set to $\phi_{m}=1$ and $\phi_{f}=1$. Let us denote the tangential and normal vectors to $\Gamma$ by

$$
\mathbf{t}=\left(\begin{array}{c}
\cos (\theta) \\
\sin (\theta)
\end{array}\right), \quad \mathbf{n}=\left(\begin{array}{c}
-\sin (\theta) \\
\cos (\theta)
\end{array}\right)
$$

Looking for a pressure solution equal to $u_{m, k}(x, y)=-x+\gamma_{k}$ in the matrix domains $\Omega_{k}, k=1$, 2 , and to $u_{f}=1-x$ in the fracture leads to

$$
\gamma_{1}=1+\frac{\sin (\theta)}{2} d_{f} \frac{\lambda_{m}}{\lambda_{f, \mathbf{n}}}, \quad \gamma_{2}=1-\frac{\sin (\theta)}{2} d_{f} \frac{\lambda_{m}}{\lambda_{f, \mathbf{n}}} .
$$


for the discontinuous hybrid-dimensional models and to

$$
\gamma_{1}=\gamma_{2}=1
$$

for the continuous hybrid-dimensional model $\left(u_{m, 1}, u_{m, 2}, u_{f}\right)$ are solutions of the corresponding stationary pressure models, see [12], [10]). We deduce that the matrix velocity is equal to

$$
\mathbf{q}_{m}=\lambda_{m}\left(\begin{array}{l}
1 \\
0
\end{array}\right)
$$

and the tangential velocity in the fracture to

$$
\mathbf{q}_{f}=d_{f} \lambda_{f} \cos (\theta) \mathbf{t}
$$

Note that the velocity fields are the same for the discontinuous and continuous hybrid-dimensional models. The transport model reduces, for all the hybrid-dimensional models presented in section 2 , to the same following system of equations which specifies our choice of the boundary and initial conditions:

$$
\left\{\begin{aligned}
\partial_{t} c_{m, k}(x, y, t)+\partial_{x} c_{m, k}(x, y, t) & =0 & & \text { on } \Omega_{k} \times(0, T), k=1,2, \\
c_{m, k}(x, y, 0) & =0 & & \text { on } \Omega_{k}, k=1,2, \\
c_{m, 1}(0, y, t) & =1 & & \text { on }\left(\frac{1}{4}, 1\right) \times(0, T), \\
c_{m, 2}(0, y, t) & =1 & & \text { on }\left(0, \frac{1}{4}\right) \times(0, T), \\
c_{m, 2}\left(x, \frac{1}{4}+x \tan (\theta), t\right) & =c_{f}(x, t) & & \text { on }(0,1) \times(0, T), \\
\partial_{t} c_{f}(x, t)+k \partial_{x} c_{f}(x, t)+\beta c_{f}(x, t) & =\beta c_{m, 1}\left(x, \frac{1}{4}+x \tan (\theta), t\right) & & \text { on }(0,1) \times(0, T), \\
c_{f}(0, t) & =1 & & \text { on }(0, T), \\
c_{f}(x, 0) & =0 & & \text { on }(0,1),
\end{aligned}\right.
$$

where we have set $\lambda_{m}=1$ and with $\beta=\frac{\sin (\theta)}{d_{f}}$ and $k=\lambda_{f} \cos ^{2}(\theta)$. It is assumed that $k>1$. This system can be integrated along the characteristics of the matrix and fracture velocity fields leading to the following analytical solution:

$$
\begin{aligned}
& c_{m, 1}(x, y, t)=\left\{\begin{array}{l}
0 \text { if } t<x, \\
1 \text { if } t>x,
\end{array}\right. \\
& c_{f}(x, t)=\left\{\begin{array}{cll}
0 & \text { if } t<\frac{x}{k}, \\
e^{-\frac{\beta}{k-1}(x-t)} & \text { if } \quad \frac{x}{k}<t<x, \\
1 & \text { if } t>x,
\end{array}\right. \\
& c_{m, 2}(x, y, t)=\left\{\begin{array}{l}
\text { if } y \in\left(0, \frac{1}{4}\right)\left\{\begin{array}{l}
0 \text { if } t<x, \\
1 \text { if } t>x,
\end{array}\right. \\
\quad \text { if } y \in\left(\frac{1}{4}, \frac{1}{4}+\tan (\theta)\right)\left\{\begin{array}{rlr}
0 & \text { if } t<x-\frac{4 y-1}{4 \tan (\theta)}, \\
c_{f}\left(\frac{4 y-1}{4 \tan (\theta)}, t+\frac{4 y-1}{4 \tan (\theta)}-x\right) & \text { if } t>x-\frac{4 y-1}{4 \tan (\theta)} .
\end{array}\right.
\end{array}\right.
\end{aligned}
$$

\subsection{Analytical solution for the equi-dimensional model}

Let us set $d=\frac{d_{f}}{\cos (\theta)}$ and denote by

$$
\widetilde{\Omega}_{f}=\left\{(x, y) \in \Omega \mid y-x \tan (\theta)-\frac{1}{4} \in\left(-\frac{d}{2}, \frac{d}{2}\right)\right\},
$$

the fracture domain, and by $\widetilde{\Omega}_{1}=\left\{(x, y) \in \Omega \mid y>\frac{d}{2}+\frac{1}{4}+x \tan (\theta)\right\}$, and $\widetilde{\Omega}_{2}=\{(x, y) \in \Omega \mid y<$ $\left.-\frac{d}{2}+\frac{1}{4}+x \tan (\theta)\right\}$ the matrix domains (see the right Figure 10). We look for a pressure solution $u_{m, k}=-x+\bar{\gamma}_{k}$ in the matrix domains $\widetilde{\Omega}_{k}, k=1,2$ and

$$
u_{f}=1-\alpha_{f}\left(\mathbf{x} \cdot \mathbf{t}-\frac{\sin (\theta)}{4}\right)+\beta_{f}\left(\mathbf{x} \cdot \mathbf{n}-\frac{\cos (\theta)}{4}\right),
$$


in the fracture domain. It results that $\alpha_{f}=\cos (\theta)$ and

$$
\beta_{f}=\sin (\theta) \frac{\lambda_{m}}{\lambda_{f, \mathbf{n}}}
$$

and

$$
\begin{aligned}
& \bar{\gamma}_{1}=1-\left(1-\frac{\lambda_{m}}{\lambda_{f, \mathbf{n}}}\right) \frac{d_{f} \sin (\theta)}{2}=\gamma_{1}-\frac{d_{f} \sin (\theta)}{2}, \\
& \bar{\gamma}_{2}=1+\left(1-\frac{\lambda_{m}}{\lambda_{f, \mathbf{n}}}\right) \frac{d_{f} \sin (\theta)}{2}=\gamma_{2}+\frac{d_{f} \sin (\theta)}{2} .
\end{aligned}
$$

We deduce that the pressure in the fracture domain is

$$
u_{f}(\mathbf{x})=1-x-\sin (\theta)\left(1-\frac{\lambda_{m}}{\lambda_{f, \mathbf{n}}}\right) d_{\Gamma}(\mathbf{x})
$$

where $d_{\Gamma}(\mathbf{x})=\mathbf{x} \cdot \mathbf{n}-\frac{\cos (\theta)}{4}$ is the distance between $\mathbf{x}$ and $\Gamma$, and that the velocity field in the matrix is

$$
\mathbf{q}_{m}=\lambda_{m}\left(\begin{array}{l}
1 \\
0
\end{array}\right)
$$

and in the fracture

$$
\mathbf{v}_{f}=-\Lambda_{f} \nabla u_{f}=\lambda_{f} \cos (\theta) \mathbf{t}-\lambda_{m} \sin (\theta) \mathbf{n}
$$

Note that $\mathbf{v}_{f}$ is exactly equal to the tangential velocity $\frac{\mathbf{q}_{f}}{d_{f}}$ of the hybrid model plus the normal velocity $\mathbf{q}_{m} \cdot \mathbf{n}=-\lambda_{m} \sin (\theta) \mathbf{n}$ of the hybrid model. In that sense, the velocity fields are the same both in the matrix and in the fracture for the equi and hybrid-dimensional models for this test case. Also remark that the mean pressure in the width of the fracture is exactly equal to $1-x$ (except at both ends of the fracture).

Let $\mathbf{v}(\mathbf{x})$ denote the velocity field equal to $\mathbf{q}_{m}$ in the matrix domains and to $\mathbf{v}_{f}$ in the fracture domain. The equi-dimensional tracer $c$ is solution of the advection equation

$$
\partial_{t} c+\operatorname{div}(c \mathbf{v})=0
$$

with initial condition $c=0$ on $\Omega$ and input condition $c=1$ on the left boundary $x=0$. It can be easily computed analytically using the method of characteristics.

\subsection{Comparisons between the equi and hybrid tracer solutions}

It is clear from Figures (11) and (13) that the hybrid-dimensional tracer model is accurate as long as the ratio of the fracture and matrix conductivities defined by $\frac{d_{f}}{L} \frac{\lambda_{f}}{\lambda_{m}}$ is large enough where $L=1$ denotes the characteristic lengh of the matrix domain. In this test case, the hybrid-dimensional tracer model provides a very accurate solution for $\frac{d_{f}}{L} \frac{\lambda_{f}}{\lambda_{m}}=10$, a rather good one for $\frac{d_{f}}{L} \frac{\lambda_{f}}{\lambda_{m}}=1$ and shows rather large differences for $\frac{d_{f}}{L} \frac{\lambda_{f}}{\lambda_{m}}=0.1$.

Geometrically, the condition of a large fracture matrix conductivity ratio $\frac{d_{f}}{L} \frac{\lambda_{f}}{\lambda_{m}}$ means that the velocity in the fracture $\mathbf{v}_{f}$ is almost parallel to the fracture $\Gamma$. More precisely, it prescribes that the angle between $\mathbf{v}_{f}$ and $\mathbf{t}$ is small compared with the angle between $\mathbf{t}$ and the line joining the bottom left point of the fracture $\left(0, \frac{1}{4}-\frac{d}{2}\right)$ to the upper right point of the fracture $\left(1, \frac{1}{4}+\tan (\theta)+\frac{d}{2}\right)$.

\subsection{Comparisons between the hybrid tracer solution and the equi-dimensional tracer solution with normal diffusion in the fracture}

In this test case, a normal diffusion term is added for the equi-dimensional tracer model in the fracture only. The objective is to investigate the amount of normal diffusion needed in the equi-dimensional 

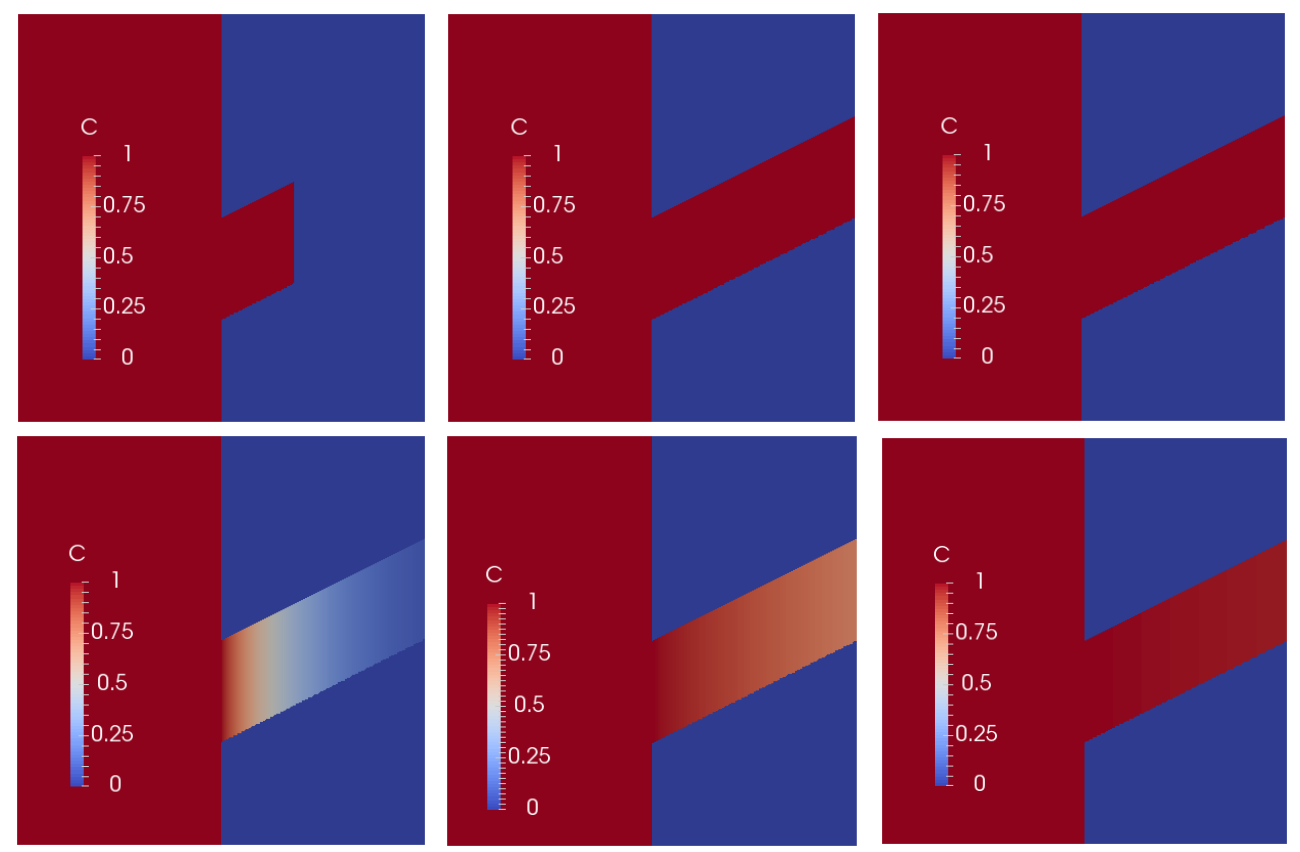

Figure 11: Comparisons of the equi-dimensional (above) and hybrid-dimensional (below) tracer analytical solutions at time $t=0.5$ for $d_{f}=0.001, \lambda_{m}=1$, and $\lambda_{f}$ taking the values from left to right 100, 1000 and 10000 .
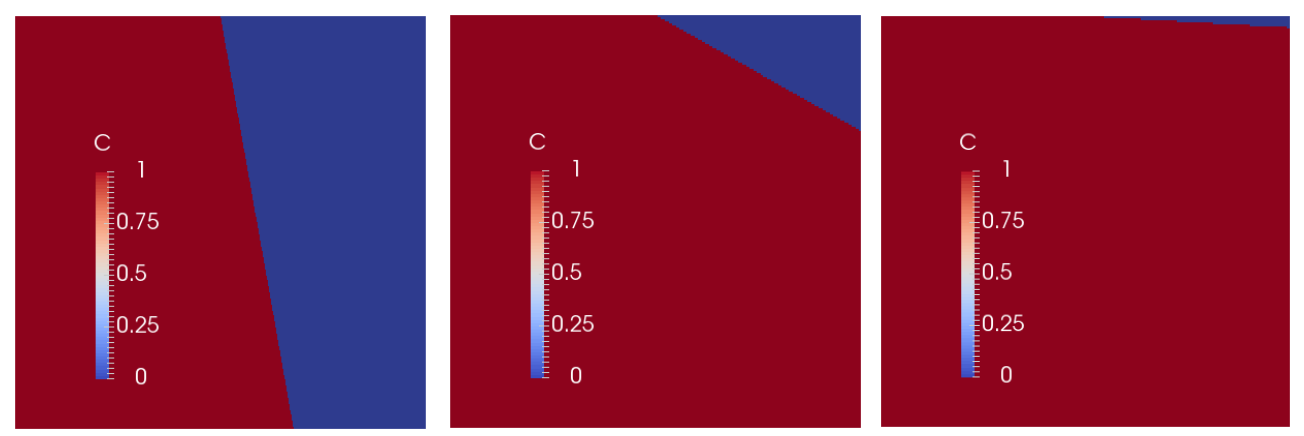

Figure 12: Tracer analytical solution in the fracture domain for the equi-dimensional model at time $t=0.5$ for $d_{f}=0.001, \lambda_{m}=1$, and $\lambda_{f}$ taking the values from left to right 100, 1000 and 10000. The fracture domain $\widetilde{\Omega}_{f}$ is mapped to $(0,1)^{2}$ using the mapping $\bar{x}=\frac{x}{L}, \bar{y}=\left(y-x \tan \theta-\frac{1}{4}+\frac{d}{2}\right) / d$

tracer model to obtain a solution close to the hybrid-dimensional tracer model without diffusion. Since in that case the analytical solution is not known, the solution of the equi-dimensional model is computed numerically using the VAG discretization and an implicit Euler time integration. We consider the above test case with $d_{f}=1$ and $\lambda_{f}=100$ which exhibits rather large differences between the hybrid and the equi-dimensional tracer models without the additional diffusion term. Let $\mathbf{n}_{\Gamma}$ denote the unit vector normal to the fracture. A diffusion term $-\operatorname{div}\left(D_{f, n} \mathbf{n}_{\Gamma} \otimes \mathbf{n}_{\Gamma} \nabla c\right)$ is added in the equi-dimensional tracer model.

It is clear from Figures 14 and 15 and the comparison with the figures of the previous subsection that the normal diffusion in the fracture provides an equi-dimensional solution much closer to the hybrid-dimensional tracer model for the parameters $\lambda_{f}=100, d_{f}=0.001$. This is expected since the hybrid-dimensional tracer model is derived assuming that the tracer concentration is constant in the width of the fractures corresponding to a high normal diffusion $D_{f, n}$ scaled by the fracture width $d_{f}$ times the normal velocity $\left|q_{m} \cdot \mathbf{n}_{\Gamma}\right|$. In our test case, this adimensionalized normal diffusion 

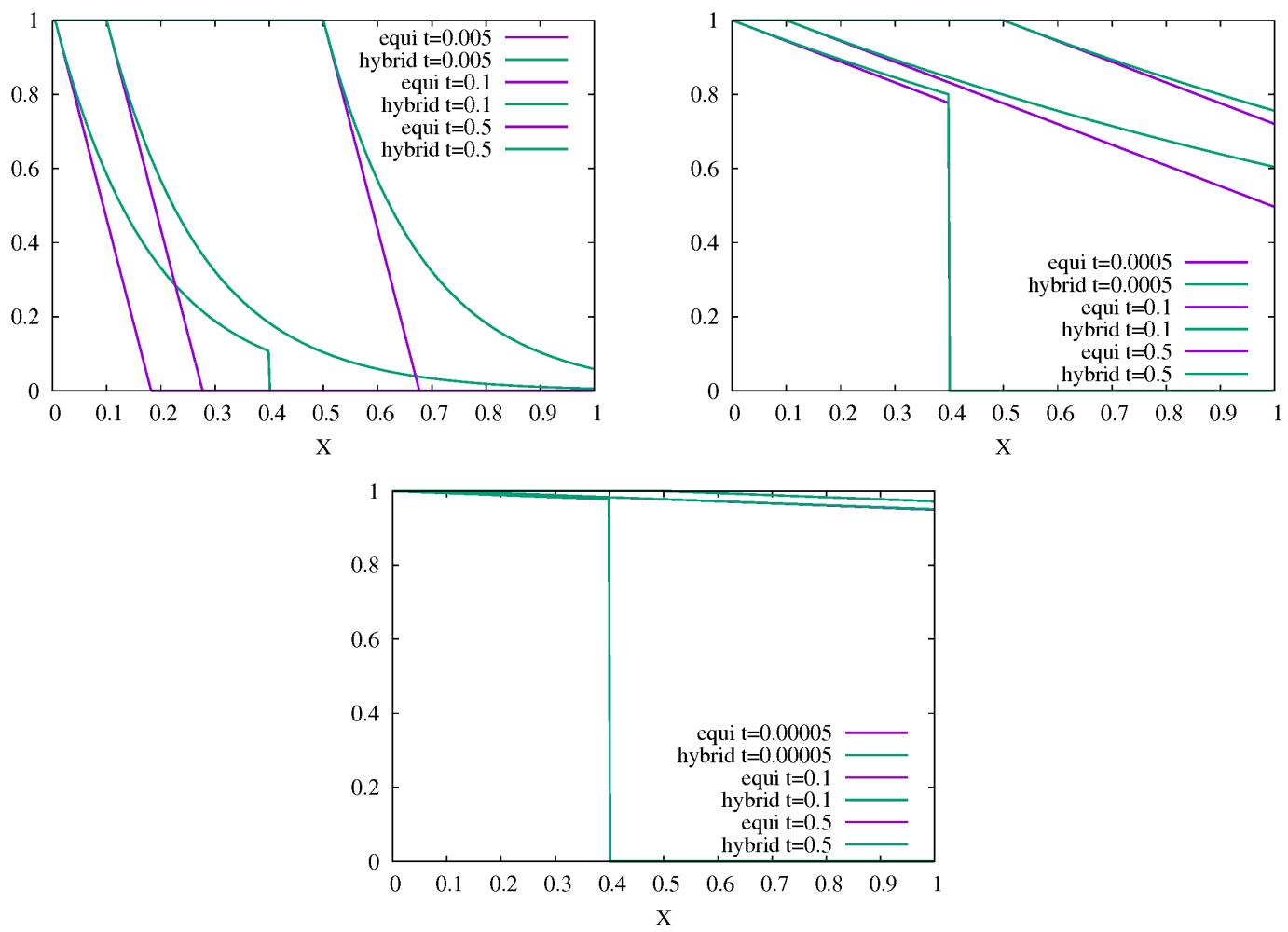

Figure 13: Comparisons of the equi and hybrid-dimensional tracer analytical solutions in the fracture at different times as a function of $x$. The value is averaged in the width of the fracture for the equidimensional model. The parameters are set to $d_{f}=0.001, \lambda_{m}=1$, and $\lambda_{f}=100,1000,10000$ from left to right.
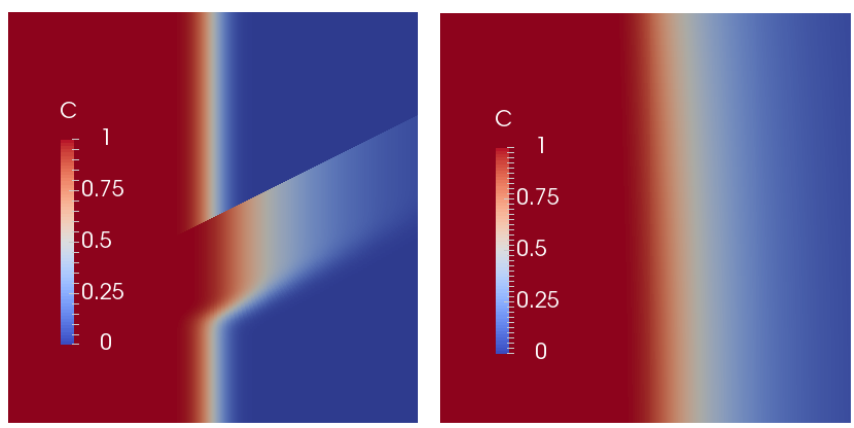

Figure 14: Tracer numerical solution at time $t=0.5$ in the matrix (left) and in the fracture (right) for the equi-dimensional model with normal diffusion in the fracture $D_{f, n}=0.001$ and for $d_{f}=0.001$, $\lambda_{m}=1$, and $\lambda_{f}=100$. The fracture domain $\widetilde{\Omega}_{f}$ is mapped to $(0,1)^{2}$ using the mapping $\bar{x}=\frac{x}{L}$, $\bar{y}=\left(y-x \tan \theta-\frac{1}{4}+\frac{d}{2}\right) / d$.

$\frac{D_{f, n}}{d_{f}\left|q_{m} \cdot \mathbf{n}_{\Gamma}\right|}$ takes the value $\sqrt{5}$ which suffices to recover a very good match between the equi and hybriddimensional models even for a rather small fracture matrix conductivity ratio $\frac{d_{f}}{L} \frac{\lambda_{f}}{\lambda_{m}}=0.1$.

\section{Model Comparison on Two-Phase Flow Test Cases}

We present in this section a series of test cases for two-phase flow through a fractured two-dimensional reservoir of geometry as shown in figure 16 . The domain $\Omega$ is of extension $(0,4) \mathrm{m} \times(0,8) \mathrm{m}$ and the 


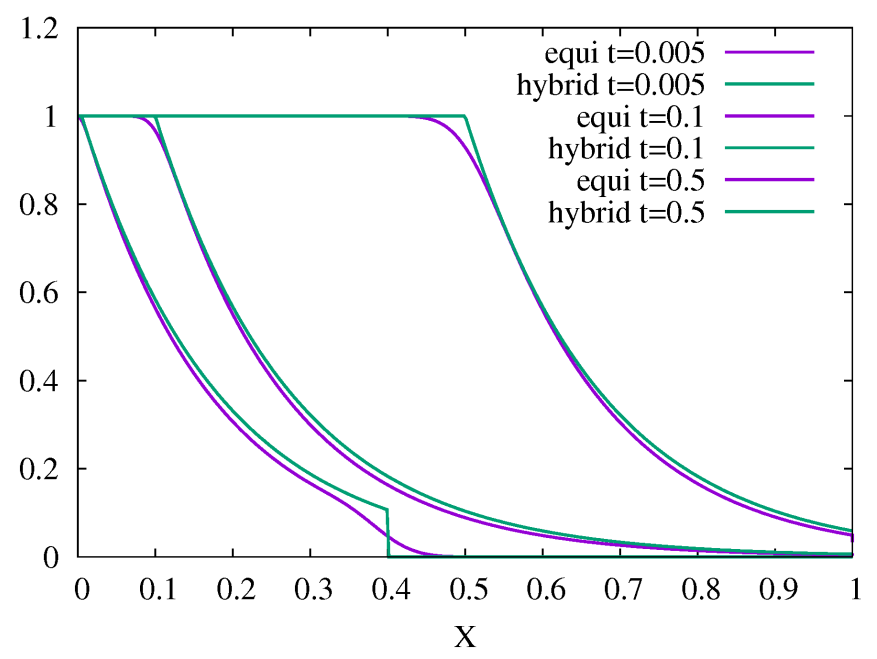

Figure 15: For $d_{f}=0.001, \lambda_{m}=1$, and $\lambda_{f}=100$, comparison of the mean tracer solution in the fracture for the equi-dimensional model (numerical) with normal diffusion in the fracture $D_{f, n}=0.001$ and the hybrid-dimensional mode (analytical).

fracture width is assumed to be constantly $d_{f}=4 \mathrm{~cm}$. We consider isotropic permeability in the matrix and in the fractures. All tests have in common that initially, the reservoir is saturated with water (density $1000 \frac{\mathrm{kg}}{\mathrm{m}^{3}}$, viscosity $0.001 \mathrm{~Pa} . \mathrm{s}$ ) and oil (density $700 \frac{\mathrm{kg}}{\mathrm{m}^{3}}$, viscosity $0.005 \mathrm{~Pa} . \mathrm{s}$ ) is injected in the bottom fracture, which is managed by imposing non-homogeneous Neumann conditions at the injection location. The oil then rises by gravity, thanks to it's lower density compared to water and by the overpressure induced by the imposed injection rate. Also, Dirichlet boundary conditions are imposed at the upper boundary of the domain. Elsewhere, we have homogeneous Neumann conditions. The following test cases present a variety of geological and physical configurations in regard to matrix and fracture permeabilities and capillary pressure curves.

We use the VAG discretization to obtain solutions for four

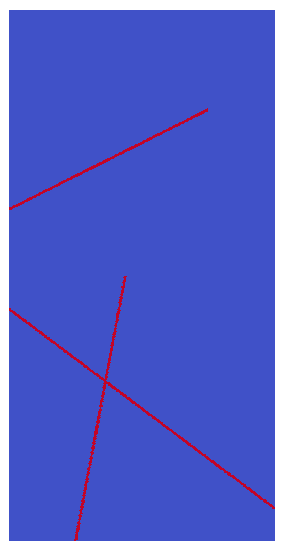
different models for this two-phase flow test case. In the first model, fractures are represented as geological structures of equal dimension as the matrix and therefore, we refer to this model as the equi-dimensional model. The second model is the hybriddimensional $m f$ nonlinear model presented in subsection 3.2 and the third and fourth models are the hybrid-dimensional $m f$ linear $m$ or $f$ upwind models presented in subsection 3.3.

The tests are driven on triangular meshes, extended to 3D prismatic meshes by adding a second layer of nodes as a translation of the original nodes in normal direction to the plane of the original 2D domain. Hence, we double the number of nodal unknowns, while keeping the number of cell and face unknowns constant (cf. table 2). In order to account for the stratification of saturation in normal direction inside the fractures, which can

Figure 16: Geometry of the reservoir under consideration. DFN in red and matrix domain in blue. $\Omega=$ $(0,4) \mathrm{m} \times(0,8) \mathrm{m}$ and $d_{f}=4 \mathrm{~cm}$. play a major role in the flow process (see below), we need at least two layers of cells in the fractures for the equi-dimensional model, to obtain valid reference solutions. Obviously, the larger number of cells for the equi-dimensional mesh is due to the need of tiny cells inside the DFN. In this regard, it is worth to mention that, with the hybrid-dimensional models, the size of fracture faces is not restricted by the fracture width, while with the equi-dimensional model, the fracture width imposes an upper bound for the size of faces between the matrix and the fracture, due to mesh regularity. However, all meshes are at 
fracture scale, here in order to focus on modelling errors. The mesh for the hybrid-dimensional models is the same, but the number of degrees of freedom differs. The supplementary degrees of freedom for the discontinuous models are located at the matrix-fracture intersections and capture the pressure discontinuities, as described in the previous section.

The discrete problem is solved implicitly, where the nonlinear system of equations occurring at each time step is solved via the Newton algorithm with relaxation. The stopping criterion is crit $_{\text {Newton }}^{\text {rel }}=$ $10^{-6}$ on the $\left(L^{1}\right)$ relative residual. For the equi-dimensional model and the hybrid-dimensional $m f$ nonlinear model, the resolution of the linear systems is performed by the GMRes solver (with stopping criterion crit $_{G M \text { Res }}^{r e l}=10^{-6}$ on the relative residual), preconditioned by CPR-AMG [28]. For both $m f$ linear models, a direct sparse linear solver is used for conveniency since this type of model would require a specific treatment of $m f$ interface equations in a CPR-AMG like preconditioner. The time loop uses adaptive time stepping, i.e. the objective for the (max per d.o.f.) change in saturation per time step, $\Delta S_{o b j}=0.5$, is given and from this the time step is deduced under the condition that it does neither exceed a given maximal time step $\Delta t_{\max }$ nor 1.2 times the time step of the previous iteration. For the equi-dimensional and hybrid-dimensional $m f$ nonlinear models, $\Delta t_{\max }=0.1$ days, and for the hybrid-dimensional $m f$ linear models, $\Delta t_{\max }=0.27$ days. Also, if at a given time iteration the Newton algorithm does not converge after 35 iterations, then the actual time step is divided by 2 and the time iteration is repeated. The number of time step failures at the end of a simulation is indicated by $\mathbf{N}_{\text {Chop }}$.

\begin{tabular}{|c|c|c|c|}
\hline Model & Nb Cells & Nb dof & Nb dof el. \\
\hline equi dim. & 22477 & 45315 & 22838 \\
\hline disc. hybrid & 16889 & 35355 & 18466 \\
\hline cont. hybrid & 16889 & 34291 & 17402 \\
\hline
\end{tabular}

Table 2: $\mathbf{N b}$ Cells is the number of cells of the mesh; $\mathbf{N b}$ dof is the number of d.o.f. (with two physical primary unknowns per d.o.f.); $\mathbf{N b}$ dof el. is the number of d.o.f. after elimination of cell unknowns without fill-in.

\subsection{Comparisons between the equi and hybrid-dimensional solutions for gravity dominated flow with zero capillary pressure}

In this test case, we neglect capillary effects by setting the capillary pressure to zero. To solve this problem, we use the matrix and fracture pressure and saturation (for, say, the non-wetting phase) as primary unknowns. The following geological configuration is considered. In the matrix domain, permeability is isotropic of 0.1 Darcy and porosity is 0.2 . In the DFN, permeability is isotropic of 100.0 Darcy and porosity is 0.4 . 

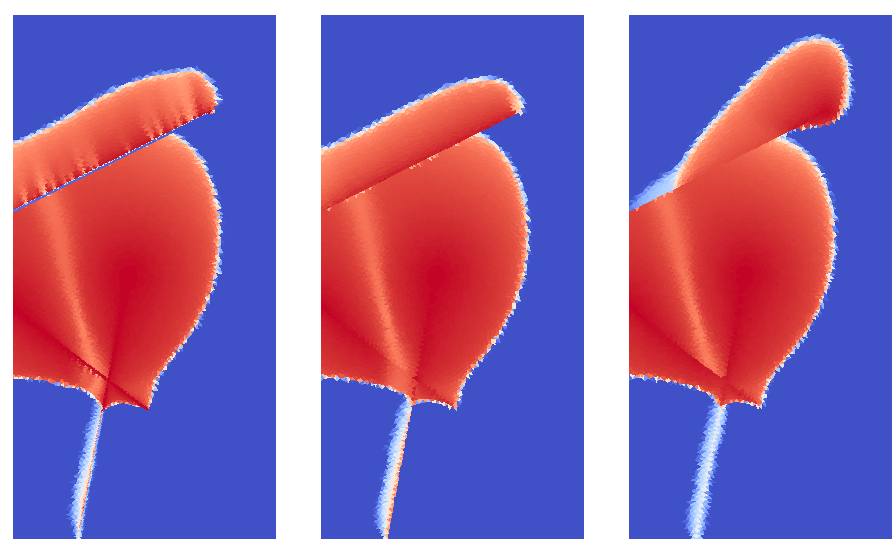

Figure 17: Comparison of the equi-dimensional, hybrid-dimensional $m f$ nonlinear, hybrid-dimensional $m f$ linear (from left to right) numerical solutions for oil saturation at final time $t=54$ days. Zero matrix and fracture capillary pressures and $m f$ permeability ratio $\lambda_{f} / \lambda_{m}=1000$.
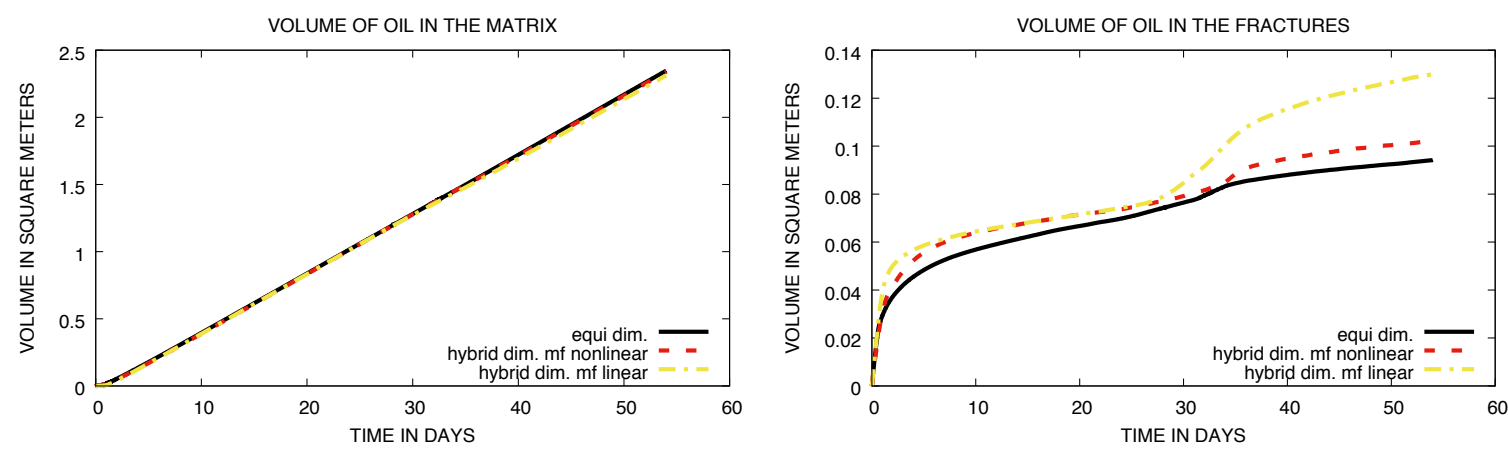

Figure 18: Comparison of the equi-dimensional and hybrid-dimensional matrix and fracture volumes occupied by oil as a function of time. Zero matrix and fracture capillary pressures and $m f$ permeability ratio $\lambda_{f} / \lambda_{m}=1000$.

\begin{tabular}{|c|c|c|c|}
\hline Model & $\mathbf{N}_{\Delta t}$ & $\mathbf{N}_{\text {Newton }}$ & $\mathbf{N}_{\text {Chop }}$ \\
\hline equi dim. & 1270 & 8927 & 71 \\
\hline hybrid $m f$ nonlinear & 907 & 5023 & 48 \\
\hline hybrid $m f$ linear & 182 & 1593 & 0 \\
\hline
\end{tabular}

Table 3: $\mathbf{N}_{\Delta t}$ is the number of successful time steps; $\mathbf{N}_{\text {Newton }}$ is the total number of Newton iterations (for successful time steps); $\mathbf{N}_{C h o p}$ is the number of time step chops. Zero matrix and fracture capillary pressures and $m f$ permeability ratio $\lambda_{f} / \lambda_{m}=1000$.

This test case shows impressively, how the incorporation of nonlinear normal fluxes at the $m f$ intersections of the hybrid-dimensional $m f$ nonlinear model allows to get much closer to the equidimensional reference solution than the hybrid-dimensional $m f$ linear models. The latter models are the same, here, since there is no saturation discontinuity due to discontinuous capillary functions. The hybrid-dimensional $m f$ nonlinear model is capable to capture the segregation of saturation inside the DFN (due to gravity, here). In this view, the supplementary d.o.f. appear as a mesh refinement at the $m f$ interfaces, that allows to reproduce the transport in normal direction to the DFN, which is not the case for the hybrid-dimensional $m f$ linear models, since there, saturations at the interfaces 
are not calculated, but fracture saturations are used for the upwinding in the nonlinear $m f$ fluxes. In the gravity dominated test case shown in figure 17, this becomes particularly important, when gravitational acceleration is in a steep angle to the fracture network, which can be observed at the upper fracture. The drawback of this feature is that we have to deal with small accumulation volumes at the $m f$ intersections, which is reflected in terms of robustness, but the hybrid-dimensional model is still much more robust than the full equi-dimensional model, even on the fine (fracture scale) meshes of these tests. The absence of capillarity, of course, emphasizes this difference between the hybriddimensional models, since at the $m f$ interfaces, the matrix does not behave as a capillary barrier (saturation does not jump) and nothing holds back the oil from leaving the DFN. Also no capillary diffusion inside the fracture prevents the gravity segregation effect in the normal direction of the fracture.

\subsection{Comparisons between the equi and hybrid-dimensional solutions for discon- tinuous capillary pressure}

The tests presented here account for capillarity. Inside the matrix domain, the capillary pressure function is given by Corey's law $p_{m}=-a_{m} \log \left(1-S_{m}^{1}\right)$. Inside the fracture network, we suppose $p_{f}=-a_{f} \log \left(1-S_{f}^{1}\right)$. To increase the robustness of the nonlinear solver and to be able to consider the degenerate case $a_{f}=0$, the choice of the phase pressures as primary unknowns is modified following [10]. At $m f$ interfacial d.o.f. $\nu_{m} \in d o f_{\mathcal{D}_{m}}^{\Gamma}$ the primary unknowns are $u^{1}$ and $\tau$ where the new unknown $\tau \in\left[0, \tau_{2}\right)$ is based on a switch of variable technique between the fracture and matrix oil saturations such that

$$
S_{f}^{2}(\tau)=\left\{\begin{array}{ll}
\tau, & \tau \in\left[0, \tau_{1}\right), \\
1-\left(\tau_{1}+\left(1-\tau_{1}\right)^{\frac{a_{f}}{a_{m}}}-\tau\right)^{\frac{a_{m}}{a_{f}}},
\end{array} S_{m}^{2}(\tau)= \begin{cases}1-(1-\tau)^{\frac{a_{f}}{a_{m}}}, & \\
\tau-\tau_{1}+1-\left(1-\tau_{1}\right)^{\frac{a_{f}}{a_{m}}}, & \tau \in\left[\tau_{1}, \tau_{2}\right),\end{cases}\right.
$$

and $u^{2}=u^{1}+p(\tau)$ with

$$
p(\tau)= \begin{cases}-a_{f} \ln (1-\tau), & \tau \in\left[0, \tau_{1}\right), \\ -a_{m} \ln \left(\tau_{1}+\left(1-\tau_{1}\right)^{\frac{a_{f}}{a_{m}}}-\tau\right), & \tau \in\left[\tau_{1}, \tau_{2}\right),\end{cases}
$$

where $\tau_{1}=1-\left(\frac{a_{f}}{a_{m}}\right)^{\frac{a_{m}}{a_{m}-a_{f}}}$ and $\tau_{2}=\tau_{1}+\left(1-\tau_{1}\right)^{\frac{a_{f}}{a_{m}}}$. It can be checked that the limit for $a_{f}=0$ and $a_{m}>0$ is well defined and such that $\tau_{1}=1, \tau_{2}=2$. At the remaining d.o.f., corresponding in our test case to homogeneous regions, the primary unknowns are chosen as the water pressure $u^{1}$ and the oil saturation.

In the degenerate case $a_{f}=0$, the derivatives of $S_{m}^{2}(\tau)$ and $p(\tau)$ with respect to $\tau$ vanish for $\tau \in(0,1)$. In order to avoid a singular Jacobian during the Newton iterates for the hybrid-dimensional $m f$ nonlinear model, a small fraction of the matrix and fracture porous volume is distributed to the $m f$ interfacial d.o.f. More precisely, we replace the accumulation terms occurring in the equations (12) for $\nu_{m} \in \operatorname{dof}_{\mathcal{D}_{m}}^{\Gamma}$ by

$$
\frac{1}{2} \sum_{K \in \mathcal{M}_{\nu_{m}}}\left|\omega_{K, \nu_{m}}\right| \phi_{K}\left(\frac{S_{m}^{\alpha}\left(\tau_{\nu_{m}}^{n}\right)-S_{m}^{\alpha}\left(\tau_{\nu_{m}}^{n-1}\right)}{\Delta t^{n}}+\frac{S_{f}^{\alpha}\left(\tau_{\nu_{m}}^{n}\right)-S_{f}^{\alpha}\left(\tau_{\nu_{m}}^{n-1}\right)}{\Delta t^{n}} .\right)
$$

It is important to note that the interfacial volumes $\omega_{K}^{\nu_{m}}, \nu_{m} \in d o f_{\mathcal{D}_{m}}^{\Gamma}$, have to be chosen small in comparison with the fracture volumes. Otherwise, the discretization would artificially widen the drain, as discussed in [10] for the continuous hybrid-dimensional model.

\subsubsection{Zero capillary pressure inside the fractures}

The geological setting is as follows. In the matrix domain, permeability is isotropic of 0.1 Darcy and porosity is 0.2 . In the DFN, permeability is isotropic of 100.0 Darcy and porosity is 0.4 . The Corey parameters are $a_{m}=1$ bar and $a_{f}=0$ bar. 

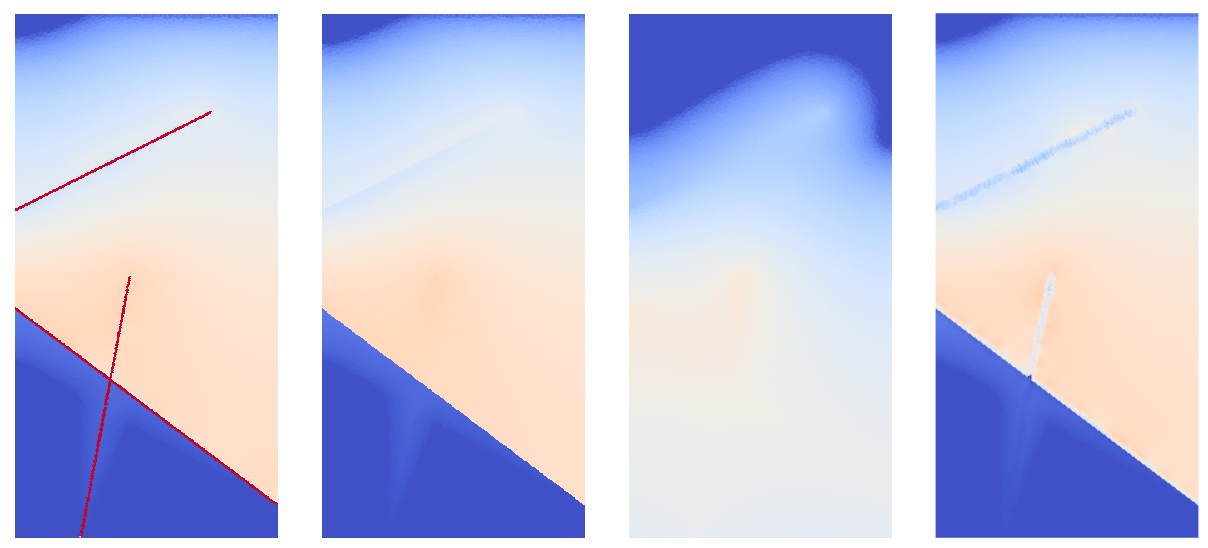

Figure 19: Comparison of the equi-dimensional, hybrid-dimensional $m f$ nonlinear, hybrid-dimensional $m f$ linear $m$ upwind and hybrid-dimensional $m f$ linear $f$ upwind (from left to right) numerical solutions for oil saturation at final time $t=54$ days. Corey parameters are $a_{m}=1$ bar, $a_{f}=0$ bar and $m f$ permeability ratio $\lambda_{f} / \lambda_{m}=1000$.
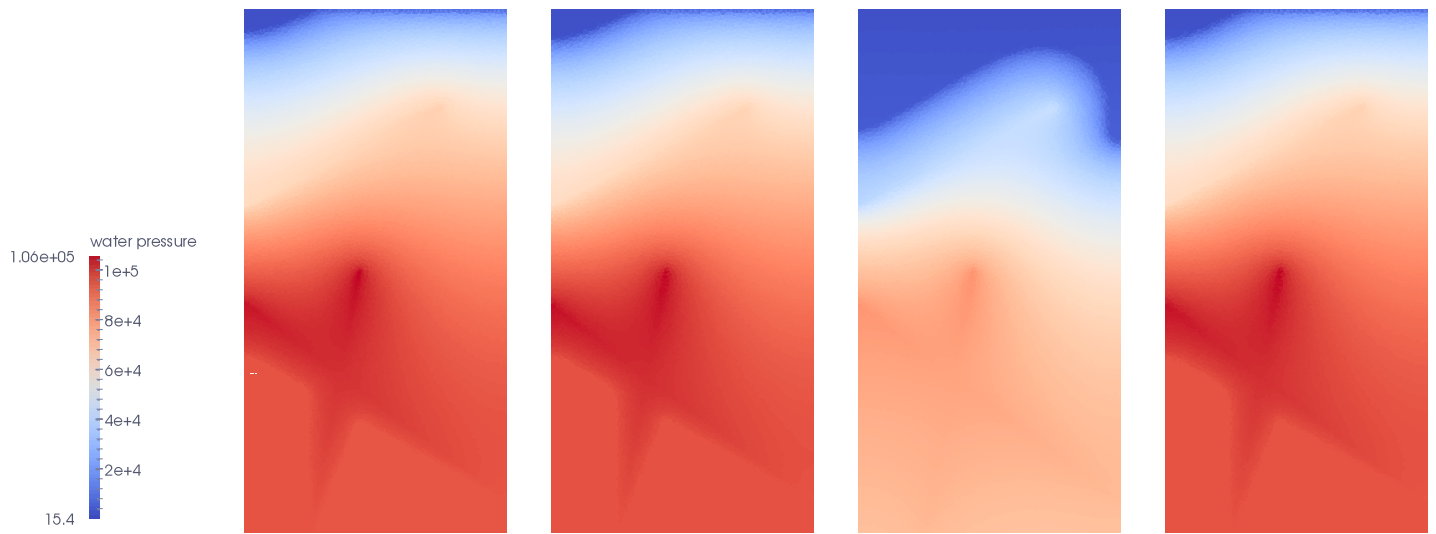

Figure 20: Comparison of the equi-dimensional, hybrid-dimensional $m f$ nonlinear, hybrid-dimensional $m f$ linear $m$ upwind and hybrid-dimensional $m f$ linear $f$ upwind (from left to right) numerical solutions for water overpressure at final time $t=54$ days. Corey parameters are $a_{m}=1$ bar, $a_{f}=0$ bar and $m f$ permeability ratio $\lambda_{f} / \lambda_{m}=1000$. 

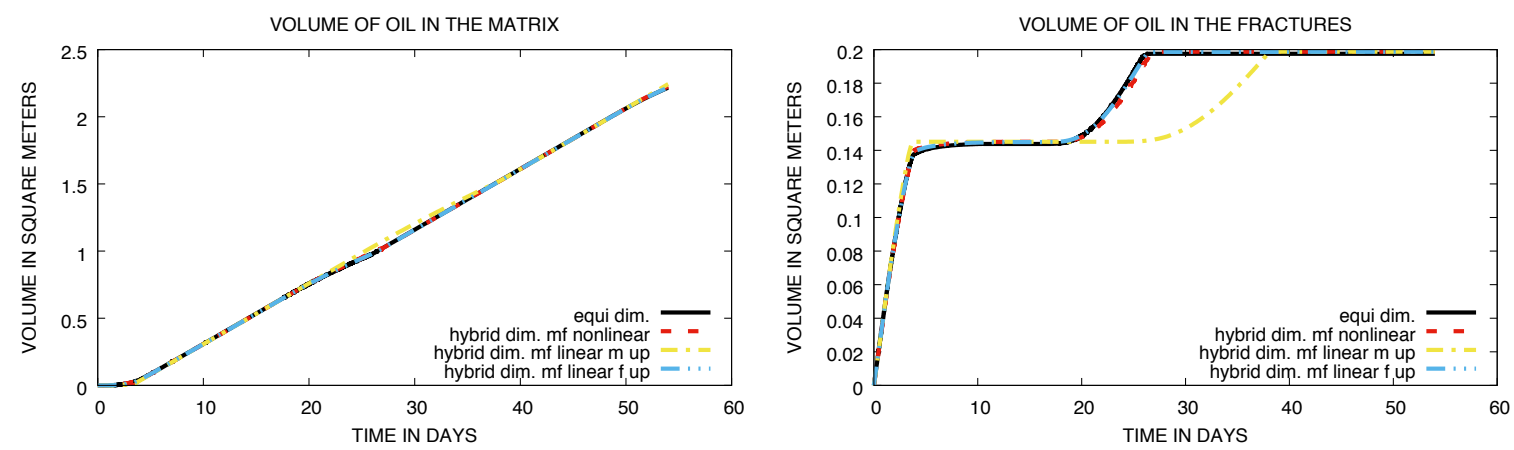

Figure 21: Comparison of the equi-dimensional and hybrid-dimensional matrix and fracture volumes occupied by oil as a function of time. Corey parameters are $a_{m}=1$ bar, $a_{f}=0$ bar and $m f$ permeability ratio $\lambda_{f} / \lambda_{m}=1000$.

\begin{tabular}{|c|c|c|c|}
\hline Model & $\mathbf{N}_{\Delta t}$ & $\mathbf{N}_{\text {Newton }}$ & $\mathbf{N}_{\text {Chop }}$ \\
\hline equi dim. & 3400 & 21529 & 336 \\
\hline hybrid $m f$ nonlinear & 3398 & 14778 & 299 \\
\hline hybrid $m f$ linear $m$ up & 510 & 3932 & 4 \\
\hline hybrid $m f$ linear $f$ up & 420 & 2799 & 0 \\
\hline
\end{tabular}

Table 4: $\mathbf{N}_{\Delta t}$ is the number of successful time steps; $\mathbf{N}_{\text {Newton }}$ is the total number of Newton iterations (for successful time steps); $\mathbf{N}_{\text {Chop }}$ is the number of time step chops. Corey parameters are $a_{m}=1$ bar, $a_{f}=0$ bar and $m f$ permeability ratio $\lambda_{f} / \lambda_{m}=1000$.

\subsection{2 drain-matrix with non-zero discontinuous capillary pressure}

The geological setting is as follows. In the matrix domain, permeability is isotropic of 0.1 Darcy and porosity is 0.2 . In the DFN, permeability is isotropic of 100.0 Darcy and porosity is 0.4 .
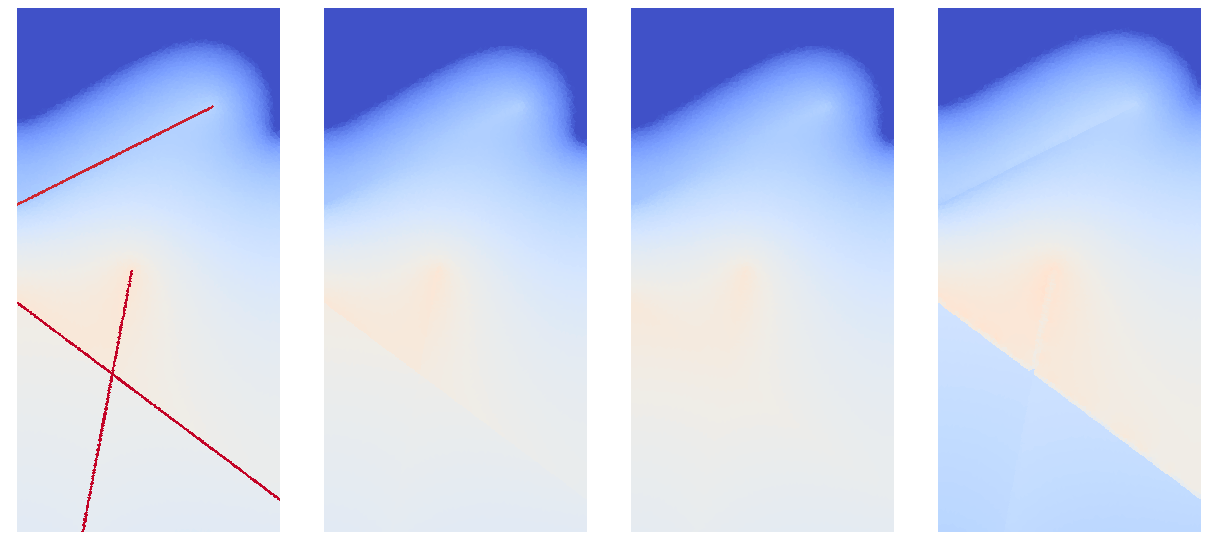

Figure 22: Comparison of the equi-dimensional, hybrid-dimensional $m f$ nonlinear, hybrid-dimensional $m f$ linear $m$ upwind and hybrid-dimensional $m f$ linear $f$ upwind (from left to right) numerical solutions for oil saturation at final time $t=54$ days. Corey parameters are $a_{m}=1$ bar, $a_{f}=0.1$ bar and $m f$ permeability ratio $\lambda_{f} / \lambda_{m}=1000$. 

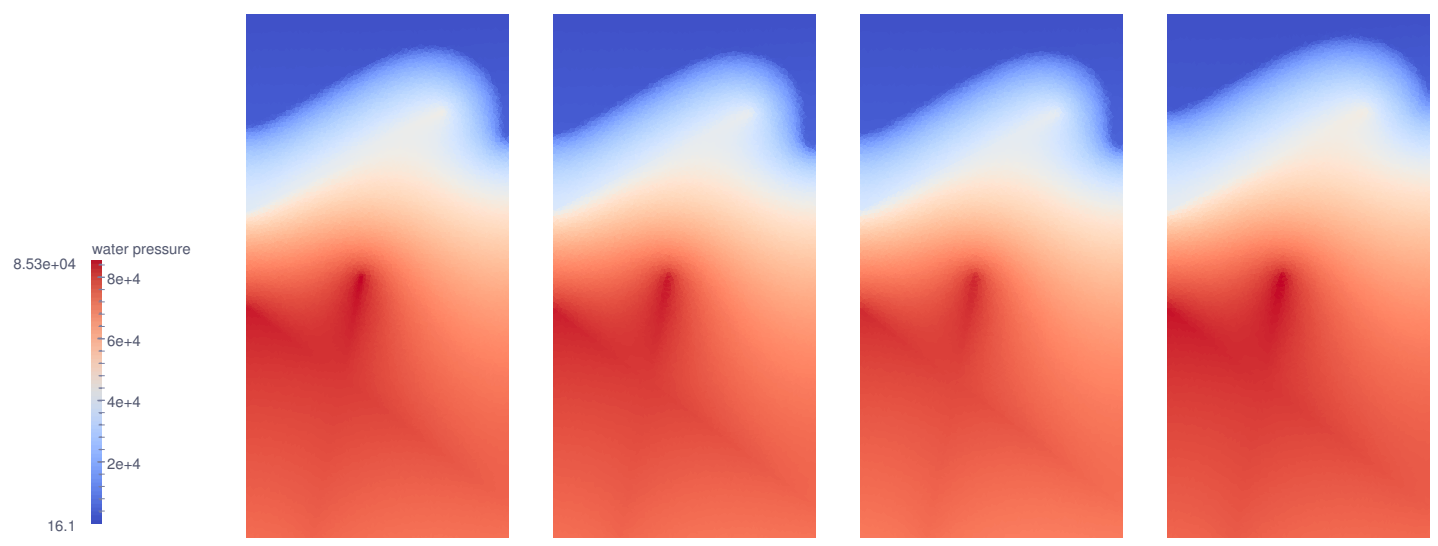

Figure 23: Comparison of the equi-dimensional, hybrid-dimensional $m f$ nonlinear, hybrid-dimensional $m f$ linear $m$ upwind and hybrid-dimensional $m f$ linear $f$ upwind (from left to right) numerical solutions for water overpressure at final time $t=54$ days. Corey parameters are $a_{m}=1$ bar, $a_{f}=0.1$ bar and $m f$ permeability ratio $\lambda_{f} / \lambda_{m}=1000$.
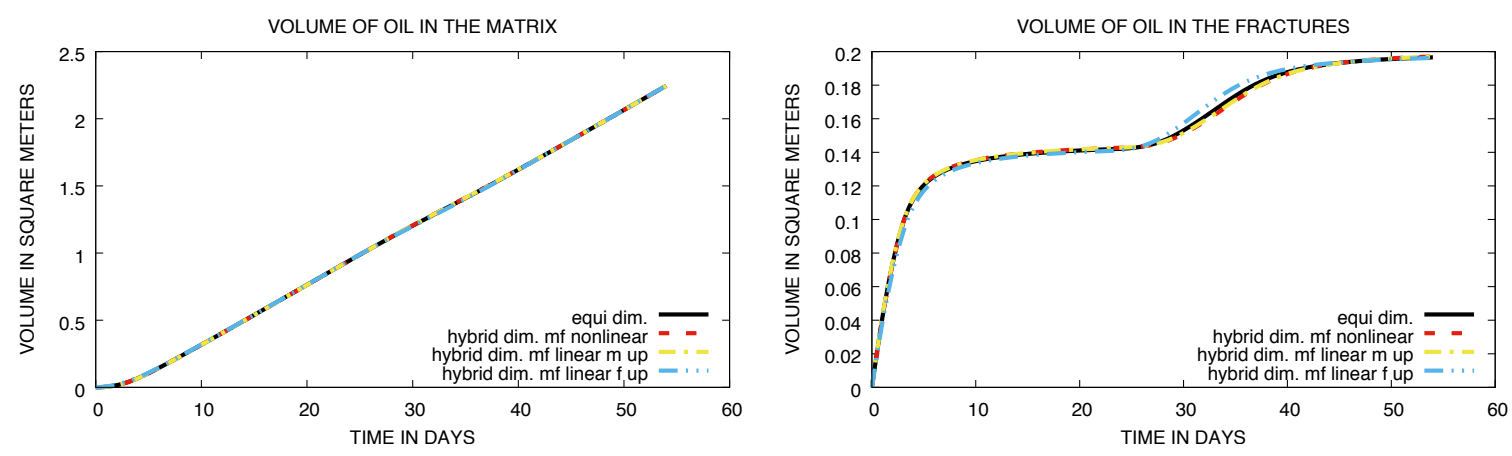

Figure 24: Comparison of the equi-dimensional and hybrid-dimensional matrix and fracture volumes occupied by oil as a function of time. Corey parameters are $a_{m}=1$ bar, $a_{f}=0.1$ bar and $m f$ permeability ratio $\lambda_{f} / \lambda_{m}=1000$.

\begin{tabular}{|c|c|c|c|}
\hline Model & $\mathbf{N}_{\Delta t}$ & $\mathbf{N}_{\text {Newton }}$ & $\mathbf{N}_{\text {Chop }}$ \\
\hline equi dim. & 589 & 2658 & 2 \\
\hline hybrid $m f$ nonlinear & 583 & 2237 & 0 \\
\hline hybrid $m f$ linear $m$ up & 255 & 1252 & 0 \\
\hline hybrid $m f$ linear $f$ up & 255 & 1227 & 0 \\
\hline
\end{tabular}

Table 5: $\mathbf{N}_{\Delta t}$ is the number of successful time steps; $\mathbf{N}_{\text {Newton }}$ is the total number of Newton iterations (for successful time steps); $\mathbf{N}_{C h o p}$ is the number of time step chops. Corey parameters are $a_{m}=1$ bar, $a_{f}=0.1$ bar and $m f$ permeability ratio $\lambda_{f} / \lambda_{m}=100$.

In the two test cases above, the fractures are filled rapidly. The behaviour of the rock matrix acting as a capillary barrier due to saturation jumps at the $m f$ interfaces lies therefore not in the focus of these tests.

The most striking observation obviously is the mismatch of the hybrid-dimensional $m f$ linear $m$ upwind solution w.r.t. the equi-dimensional reference solution, exhibited in figure 19, which also shows that the solution of the hybrid-dimensional $m f$ linear models depends strongly on the choice of the $m f$ fluxes given either by (13) or by (15). While (13) respects the saturation jumps at the $m f$ interfaces, which prevents oil from penetrating into the matrix for fracture which are not yet saturated with oil, 
the barrier effect of the matrix is reduced compared with the equi-dimensional or hybrid-dimensional $m f$ nonlinear solutions at the $m f$ interfaces saturated with oil. On the other hand, for (15), saturation jumps due to discontinuous capillary pressure curves at the $m f$ interfaces are not treated, which lets oil penetrate into the matrix even when the fracture is not yet saturated with oil, however the barrier effect of the matrix is better reproduced for fully saturated fractures and the solution seems to converge to the reference solution, though the matrix cells touching the fractures yield considerable errors. The hybrid-dimensional $m f$ nonlinear model does not suffer from these difficulties since it distinguishes between the unknowns at the interfaces capturing the saturation jumps and the unknowns inside the fracture, providing for this test case a zero capillary pressure as expected.

Figures 19, 22, 21 and 24 reveal that the matching of equi-dimensional and hybrid-dimensional $m f$ linear $m$ upwind solutions can be enhanced by adding capillarity in the DFN. More precisely, we note that the latter solution changes insignificantly, but the equi-dimensional solution changes towards the hybrid-dimensional $m f$ linear $m$ upwind solution. Capillarity has a diffusive effect and smoothens out the stratification in the DFN, which agrees better with the hybrid-dimensional approach of averaging physical quantities over the fracture width. We observe that the solution for the hybrid-dimensional $m f$ nonlinear acts as the solution of the equi-dimensional model, whereas the hybrid-dimensional $m f$ linear $f$ upwind model does not recover the flow in the lower rock matrix accurately. In this sense, the test case underlines the importance of treating saturation jumps at the $m f$ interfaces for discontinuous capillary pressure curves, even when the fractures are rapidly filled with oil.

\subsection{Comparisons between the equi and hybrid-dimensional solutions for discon- tinuous capillary pressure at the matrix-fracture interfaces and an upper fracture-barrier}

In the matrix domain, permeability is isotropic of 0.1 Darcy and porosity is 0.2 . The two lower fractures are drains of isotropic permeability 100.0 Darcy and porosity 0.4. In the upper fracture, permeability is isotropic of 0.001 Darcy and porosity is 0.2. Note that the continuous hybrid-dimensional model does not incorporate a normal permeability in the DFN. We conducted the test case also for this model and observed, as expected, the unability to reproduce the barrier behaviour of the upper fracture. The Corey parameters are $a_{m}=1$ bar, $a_{\text {barrier }}=100$ bar and $a_{\text {drain }}=0.1$ bar.
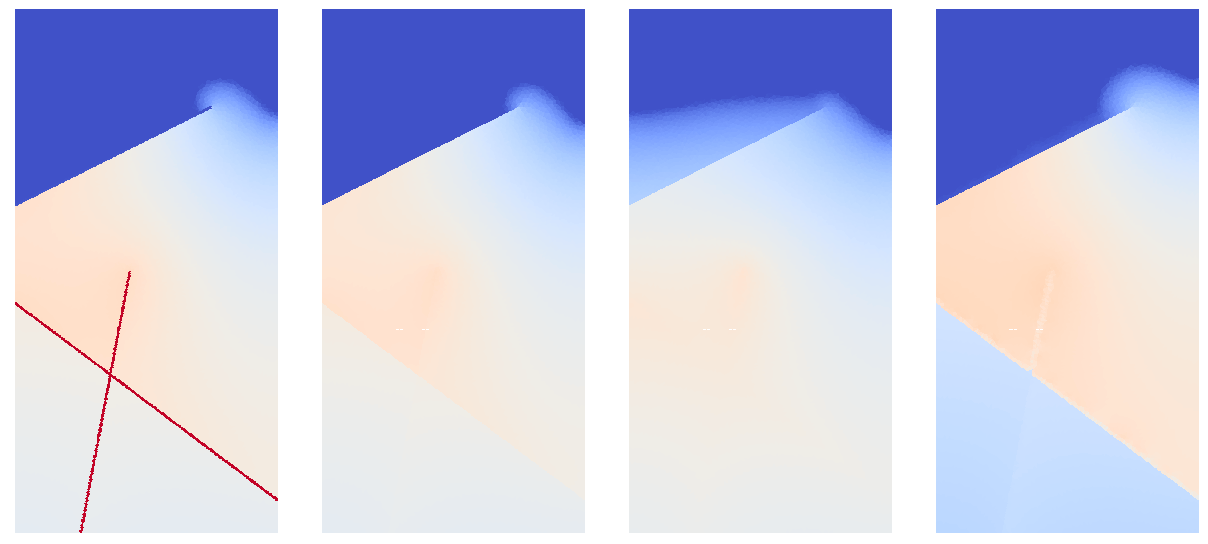

Figure 25: Comparison of the equi-dimensional, hybrid-dimensional $m f$ nonlinear, hybrid-dimensional $m f$ linear $m$ upwind and hybrid-dimensional $m f$ linear $f$ upwind (from left to right) numerical solutions for oil saturation at final time $t=54$ days. Corey parameters are $a_{m}=1$ bar, $a_{\text {barrier }}=100$ bar, $a_{\text {drain }}=0.1$ bar and $m f$ permeability ratios $\lambda_{\text {barrier }} / \lambda_{m}=0.01$ and $\lambda_{\text {drain }} / \lambda_{m}=1000$. 

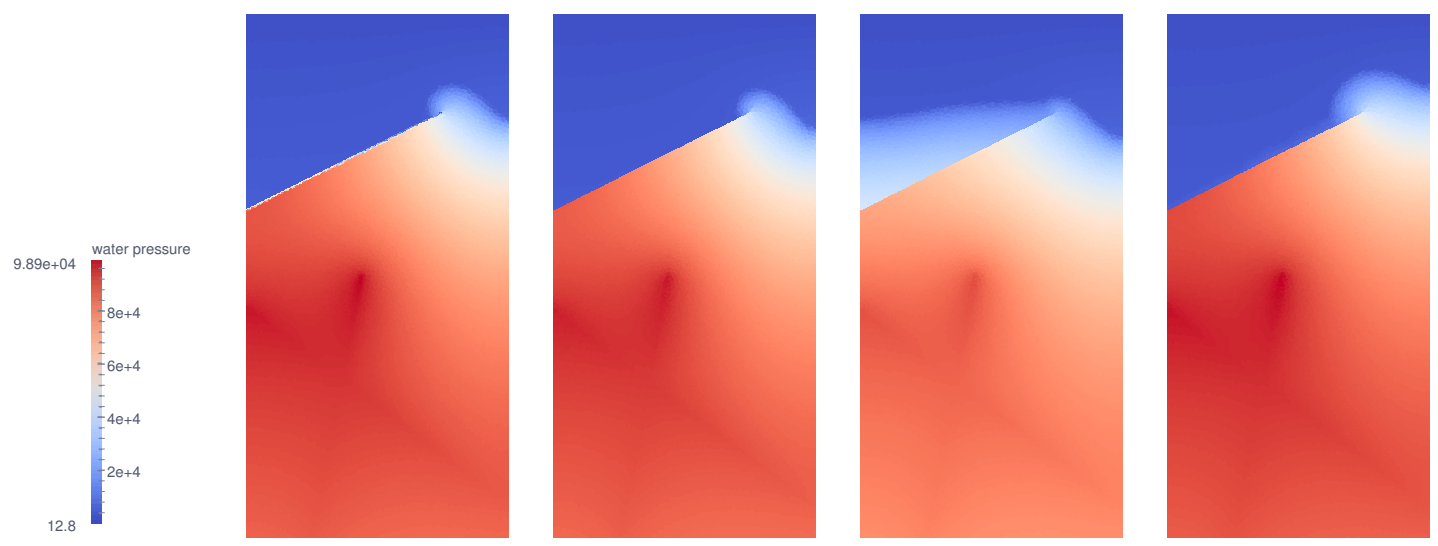

Figure 26: Comparison of the equi-dimensional, hybrid-dimensional $m f$ nonlinear, hybrid-dimensional $m f$ linear $m$ upwind and hybrid-dimensional $m f$ linear $f$ upwind (from left to right) numerical solutions for water overpressure at final time $t=54$ days. Corey parameters are $a_{m}=1$ bar, $a_{\text {barrier }}=100$ bar, $a_{\text {drain }}=0.1$ bar and $m f$ permeability ratios $\lambda_{\text {barrier }} / \lambda_{m}=0.01$ and $\lambda_{\text {drain }} / \lambda_{m}=1000$.
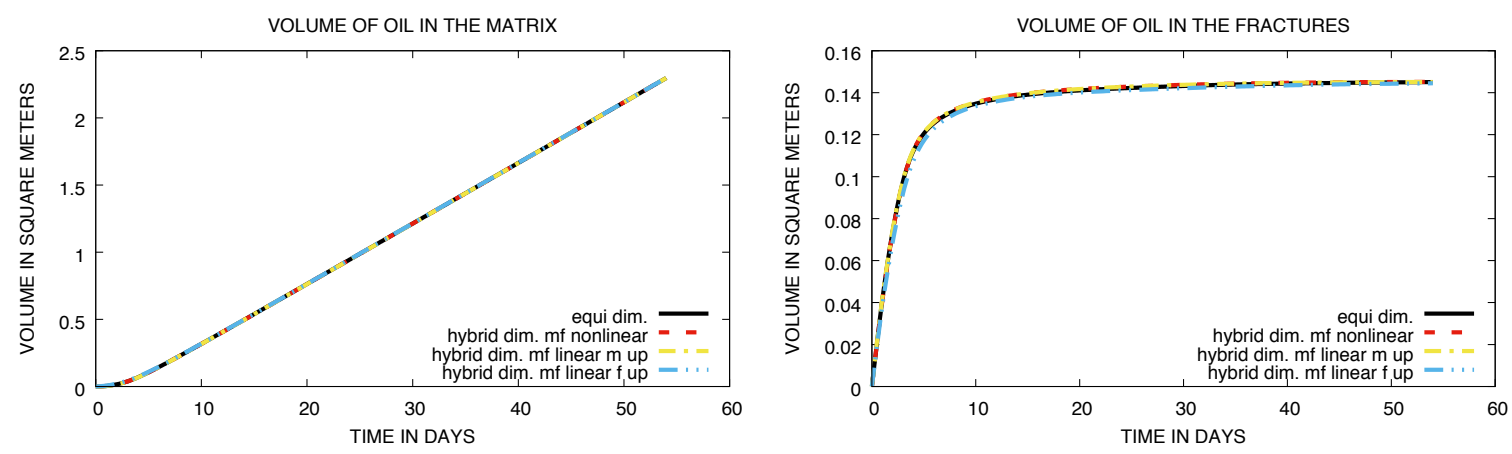

Figure 27: Comparison of the equi-dimensional and hybrid-dimensional matrix and fracture volumes occupied by oil as a function of time. Corey parameters are $a_{m}=1$ bar, $a_{\text {barrier }}=100$ bar, $a_{\text {drain }}=0.1$ bar and $m f$ permeability ratios $\lambda_{\text {barrier }} / \lambda_{m}=0.01$ and $\lambda_{\text {drain }} / \lambda_{m}=1000$.

\begin{tabular}{|c|c|c|c|}
\hline Model & $\mathbf{N}_{\Delta t}$ & $\mathbf{N}_{\text {Newton }}$ & $\mathbf{N}_{\text {Chop }}$ \\
\hline equi dim. & 607 & 2493 & 10 \\
\hline hybrid $m f$ nonlinear & 602 & 2030 & 7 \\
\hline hybrid $m f$ linear $m$ up & 255 & 1252 & 0 \\
\hline hybrid $m f$ linear $f$ up & 255 & 1176 & 0 \\
\hline
\end{tabular}

Table 6: $\mathbf{N}_{\Delta t}$ is the number of successful time steps; $\mathbf{N}_{\text {Newton }}$ is the total number of Newton iterations (for successful time steps); $\mathbf{N}_{C h o p}$ is the number of time step chops. Corey parameters are $a_{m}=1$ bar $a_{\text {barrier }}=100$ bar, $a_{\text {drain }}=0$ bar and $m f$ permeability ratios $\lambda_{\text {barrier }} / \lambda_{m}=0.01$ and $\lambda_{\text {drain }} / \lambda_{m}=$ 1000 .

In this test case, we study the presence of a fracture, which acts as a barrier, both by its low permeability and by its high capillarity compared to the rock matrix. In this case, the sign of the matrix-fracture oil saturation jump $S_{m}^{1}\left(\gamma_{\mathfrak{a}} p_{m}\right)-S_{f}^{1}\left(\gamma_{\mathfrak{a}} p_{m}\right)$ at the $m f$ interfaces is non negative. For the $m f$ linear models, mass transfer of oil from the matrix to the barrier is overestimated, since in this direction, saturation jumps are not accounted for. Consequently, the assumption of constant saturation 
accross the fracture for these models leads to an overestimation of oil leaving the barrier, and this overestimation is most severe for the $m$ upwind model, which takes into account saturation jumps for fluxes directed from the fracture to the matrix. Again, the hybrid-dimensional $m f$ nonlinear model does not suffer from this difficulty since it provides mass transport that passes by the $m f$ interfaces and takes into account the saturation jumps.

\section{Three dimensional Two-Phase Flow Test Case}

In this section, we present a test case, where flow in a full 3D matrix is coupled with flow in a 2D fracture network. We compare the output of VAG simaltions for the hybrid dimensional $m f$ nonlinear model on three tetrahedral meshes of different mesh size. The refinement is done in the neighbourhood of the DFN.

The geometry of the domain is illustrated in figure 28. The domain consists of a matrix domain of extension $100 \mathrm{~m} \times 100 \mathrm{~m} \times 100 \mathrm{~m}$ and of anisotropic permeability, which is of 0.1 Darcy in $x$ and in $y$ direction and 0.01 Darcy in $z$ direction. The fracture network is assumed to be of constant aperture $d_{f}=1 \mathrm{~cm}$ and of isotropic permeability 10 Darcy. Matrix porosity is 0.2 and fracture porosity is 0.4 . The matrix capillary pressure - saturation relation is given by $p_{m}=-a_{m} \log \left(1-S_{m}^{1}\right)$, with $a_{m}=1$ bar. Inside the fracture network, we suppose $p_{f}=0$.

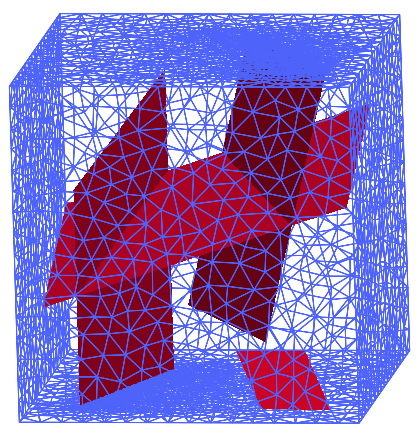

Figure 28: Geometry of the reservoir under consideration. DFN in red and matrix domain in blue wireframe. $\Omega=100 \mathrm{~m} \times 100 \mathrm{~m} \times 100 \mathrm{~m}$ and $d_{f}=1 \mathrm{~cm}$.

At initial time, the reservoir is fully saturated with water. Then, oil is injected from below, which is managed by imposing Dirichlet conditions at the bottom and at the top of the reservoir. We also impose 20 bar of over-pressure w.r.t. the hydrostatic distribution of pressure. The oil then mounts by gravity, thanks to it's less density compared to water, and by the pressure potential.

The tests are driven on tetrahedral meshes. The parameters for the numerical resolution are as in the test cases of the previous section : crit $_{\text {Newton }}^{\text {rel }}=10^{-6}$, rrit $_{G M R e s}^{r e l}=10^{-6}, \Delta S_{o b j}=0.5$. The simulation of the flow covers the period of one year. the maximal time step size is $\Delta t_{\max }=0.1$ days. 

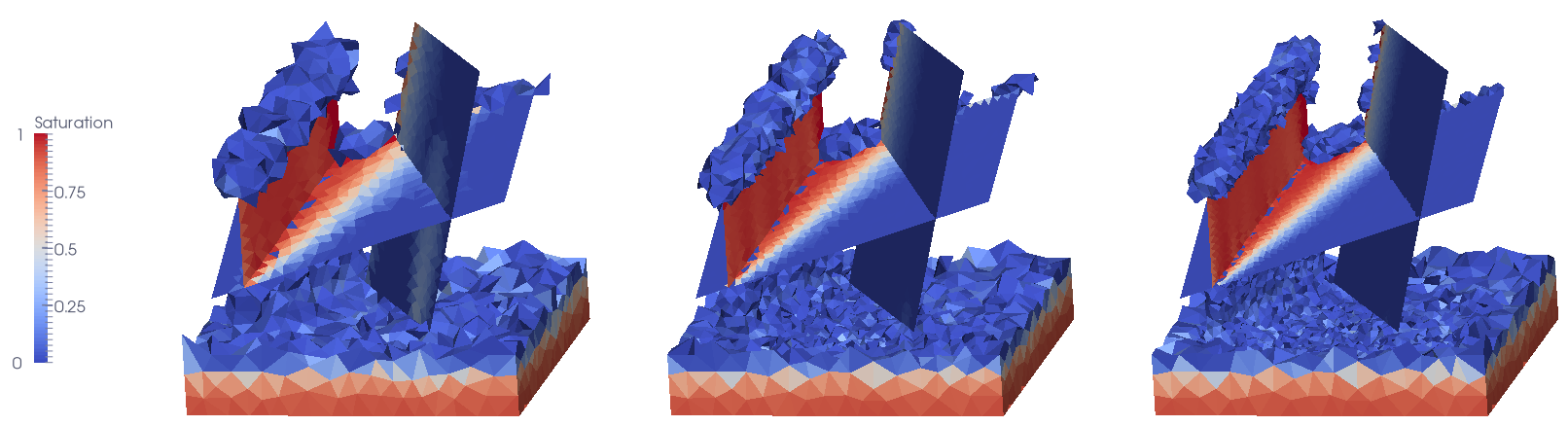

Figure 29: Comparison of the solution for oil saturation at final time $t=360$ days. Coarsest to finest mesh from left to right.
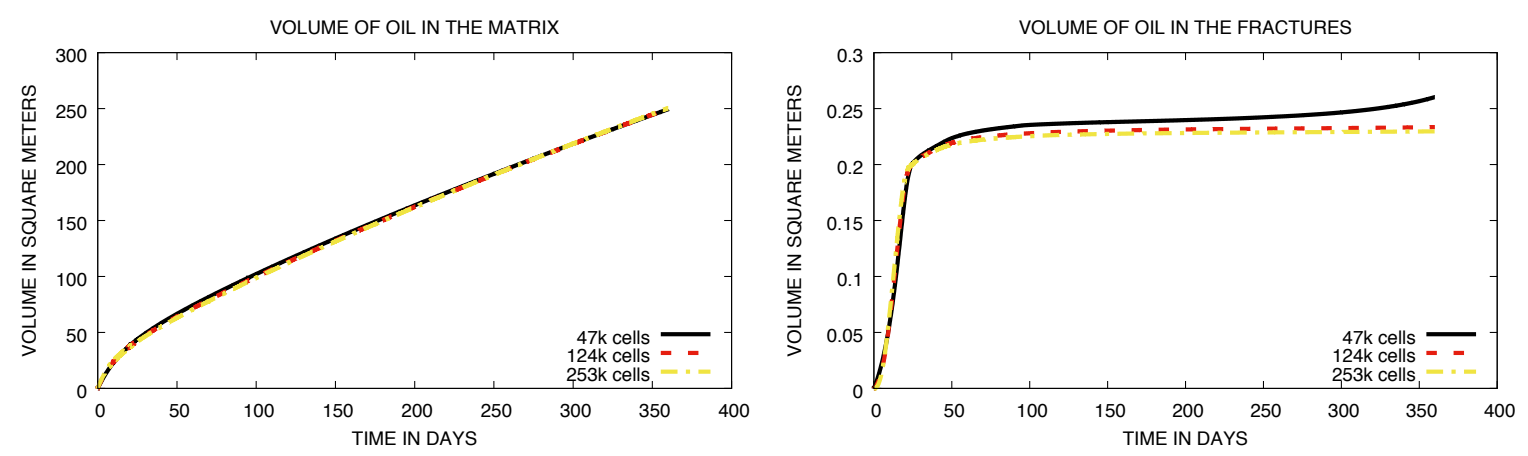

Figure 30: Comparison of the matrix and fracture volumes occupied by oil as a function of time for different meshes.

\begin{tabular}{|c|c|c|c|c|c|c|c|}
\hline Nb Cells & Nb dof & Nb dof el. & $\mathbf{N}_{\Delta t}$ & $\mathbf{N}_{\text {Newton }}$ & $\mathbf{N}_{\text {GMRes }}$ & $\mathbf{N}_{\text {Chop }}$ & CPU[s] \\
\hline 47670 & 62763 & 15093 & 3779 & 11078 & 533705 & 6 & 21180 \\
\hline 124245 & 159744 & 35499 & 4043 & 13314 & 1039288 & 19 & 93428 \\
\hline 253945 & 321670 & 67725 & 4566 & 17900 & 1518968 & 38 & 229604 \\
\hline
\end{tabular}

Table 7: $\mathbf{N b}$ Cells is the number of cells of the mesh; $\mathbf{N b}$ dof is the number of d.o.f. (with two physical primary unknowns per d.o.f.); $\mathbf{N b}$ dof el. is the number of d.o.f. after elimination of cell unknowns without fill-in. $\mathbf{N}_{\Delta t}$ is the number of successful time steps; $\mathbf{N}_{\text {Newton }}$ is the total number of Newton iterations (for successful time steps); $\mathbf{N}_{\text {Chop }}$ is the number of time step chops.

For the carsest mesh, there is an error due to large matrix cells, which let oil pass between two disconnected fractures too rapidely. However, in the region where flow is driven by the connected DFN, the solution matches well with the solution obtained on the fine mesh. We also observe a good agreement between the two solutions on the finer meshes.

Table 7 reveils a significant gain in efficiency when passing from a finer to a coarser mesh. This indicates what a powerful tool hybrid-dimensional modelling is. In fact, it would be infeasible to derive an equi-dimensional solution for this test case. 


\section{Conclusion}

The hybrid-dimensional models for two-phase flow through fractured porous media with pressure discontinuities at the matrix-fracture $(m f)$ interfaces introduced here account for network of fractures acting both as drains or barriers, general and discontinuous capillary pressures, and gravity forces. It is compared with the equi-dimensional model on various tracer and two-phase flow test cases. For the tracer problem with a fracture dividing the matrix domain, analytical solutions for different model parameters have been derived, both, for the hybrid- and equi-dimensional models. It turned out that the hybrid-dimensional models lose in precision with decreasing conductivity ratios $\frac{d_{f} \lambda_{f}}{L \lambda_{m}}$, which corresponds to steepening the angle between the equi-dimensional velocity inside the fracture and the fracture tangential directions. Then, it has been shown that adding a small normal diffusion, of the order of the normal convective flux divided by the fracture width or larger, in the fracture drives the equi-dimensional solution very close to the hybrid-dimensional solution. It was expected since the averaging procedure in the derivation of the reduced models corresponds to adding a diffusion in the normal direction inside the fracture network. The Vertex Aproximate Gradient (VAG) scheme, as introduced in [12] for the single-phase stationary hybrid-dimensional model, has been presented in a finite volume formulation for the two-phase flow models. The VAG scheme is used to compare the numerically derived solutions for the hybrid-dimensional $m f$ nonlinear, the hybrid-dimensional $m f$ linear $m$ upwind and the hybrid-dimensional $m f$ linear $f$ upwind models for a $2 \mathrm{D}$ flow process through a fractured reservoir to a reference solution given by the equi-dimensional model (full model with fractures represented as heterogeneous layers). Hereby, a variety of geological and physical configurations in regard to matrix and fracture permeabilities and capillary pressure curves is considered. Since the stratification in normal direction inside the fractures can play a major role, it is worth to mention that more than one layer of fracture cells is necessary in order to get valid reference solutions. The hybrid-dimensional $m f$ linear $m$ upwind model can be seen as an extension of the continuous hybrid-dimensional model introduced in [9] to a hybrid-dimensional discontinuous pressure model. We observed that these models produce solutions, which are almost indistiguishable, which led us to omit the results for the continuous hybrid-dimensional model. In terms of robustness, the test cases show that the hybrid-dimensional $m f$ linear models have a clear advantage. Yet, the hybrid-dimensional $m f$ nonlinear model still is much more robust than the equi-dimensional model. Moreover, for fracture matrix conductivity ratios $\frac{d_{f} \lambda_{f}}{L \lambda_{m}}>10$, the equi-dimensional model is impracticable. In the first test case, gravitational segregation has a major influence on the global flow behaviour. This effect cannot be reproduced by the hybrid-dimensional $m f$ linear models, with single unknowns for the saturation at the DFN. The supplementary information on the saturations at the $m f$ interfaces used in the $m f$ mass exchange fluxes of the hybrid-dimensional $m f$ nonlinear model enables us to capture gravitational segregation inside the DFN. Furthermore, in the examples given with capillary pressure in the matrix domain, it appears that the hybrid-dimensional $m f$ nonlinear model, using both interface and average capillary pressure unknowns in the fracture, restores the forces due to the capillary pressure difference between the $m f$ interfaces and the interior of the fractures, whereas for the hybrid-dimensional $m f$ linear models, the capillary pressure is taken constant accross the fractures, for the construction of the $m f$ mass exchange fluxes. These features make the $m f$ nonlinear model much more precise on the transport accross the DFN compared with the $m f$ linear models which cannot both capture the saturation jump at the interface and provide a good approximation of the capillary pressure inside the fracture. In the barrier test case presented here, we see again that the simplified $m f$ mass exchange of the hybrid-dimensional $m f$ linear models leads to false solutions, whereas the hybrid-dimensional $m f$ nonlinear model performs well, both, in terms of accuracy and robustness. In any case, we observed a significant gain in precision for the hybrid-dimensional $m f$ nonlinear solution w.r.t. the equi-dimensional reference solution, compared to the hybrid-dimensional $m f$ linear solutions. Finally, a 3D-matrix-2D-fracture test case is presented to compare hybrid-dimensional $m f$ nonlinear solutions obtained with the VAG scheme on three tetrahedral meshes of different mesh size. 
Acknowledgements: The authors would like to thank Total SA for its financial support and for allowing the publication of this work.

\section{References}

[1] Ahmed, R., Edwards, M.G., Lamine, S., Huisman, B.A.H., Control-volume distributed multi-point flux approximation coupled with a lower-dimensional fracture model, J. Comp. Physics, 462-489, Vol. 284, 2015.

[2] Ahmed, E., M., Jaffré, J., Roberts, J. E., A reduced fracture model for two-phase flow with different rock types, Mathematics and Computers in Simulation, 7, pp. 49-70, 2017.

[3] Alboin, C., Jaffré, J., Roberts, J., Serres, C.: Modeling fractures as interfaces for flow and transport in porous media. Fluid flow and transport in porous media 295, 13-24, 2002.

[4] Finite volume approximation of a diffusion-dissolution model and application to nuclear waste storage, Angelini, O., Chavant, C., Chénier, E., Eymard, R., Granet, S. Mathematics and Computers in Simulation, 81, pp. 2001-2017, 2011.

[5] D'Angelo, C., Scotti, A.: A mixed finite element method for Darcy flow in fractured porous media with non-matching grids. ESAIM Mathematical Modelling and Numerical Analysis 46,2, 465-489, 2012 .

[6] Angot, P., Boyer, F., Hubert, F. Asymptotic and numerical modelling of flows in fractured porous media, M2AN, 43,2, pp; 239-275, 2009.

[7] Antonietti, P.F., Formaggia, L., Scotti, A., Verani, M., Verzotti, N. Mimetic Finite Difference Approximation of flows in Fractured Porous Media, M2AN, 50, pp. 809-832, 2016.

[8] Bogdanov, I., Mourzenko, V., Thovert, J.-F., Adler, P. M., Two-phase flow through fractured porous media, Physical Review E 68, 026703, 2003.

[9] Brenner, K., Groza, M., Guichard, C., Masson, R. Vertex Approximate Gradient Scheme for Hybrid-Dimensional Two-Phase Darcy Flows in Fractured Porous Media. ESAIM Mathematical Modelling and Numerical Analysis, 49, pp. 303-330, 2015.

[10] Brenner, K., Groza, M., Jeannin, L., Masson, R., Pellerin, J. Immiscible two-phase Darcy flow model accounting for vanishing and discontinuous capillary pressures: application to the flow in fractured porous media, Computational Geosciences, online july 2017.

[11] Brenner, K., Groza, M., Guichard, C., Lebeau, G. and Masson, R. Gradient discretization of Hybrid-Dimensional Darcy Flows in Fractured Porous Media. Numerische Mathematik, 134,3, pp. 569-609, 2016.

[12] K. Brenner; J. Hennicker; R. Masson; P. Samier. Gradient discretization of hybrid-dimensional Darcy flow in fractured porous media with discontinuous pressures at matrix-fracture interfaces IMA Journal of Numerical Analysis, 37,3, pp. 1551-1585, 2016.

[13] Droniou, J., Hennicker, J., Masson, R., Numerical analysis of a two-phase flow discrete fracture model, https://arxiv.org/abs/1612.07373. Submitted

[14] Eymard, R., Guichard, C., Herbin, R. Small-stencil 3D schemes for diffusive flows in porous media. ESAIM: Mathematical Modelling and Numerical Analysis, 46, pp. 265-290, 2010.

[15] Eymard, R., Gallouët, T., Herbin, R.: Discretization of heterogeneous and anisotropic diffusion problems on general nonconforming meshes SUSHI: a scheme using stabilisation and hybrid interfaces. IMA Journal of Numerical Analysis, 30,4, pp. 1009-1043, 2010. 
[16] R. Eymard, R. Herbin, C. Guichard, R. Masson, Vertex centered discretization of compositional multiphase darcy flows on general meshes. Comp. Geosciences, 16, pp. 987-1005, 2012.

[17] R. Eymard, C. Guichard, R. Herbin, R. Masson, Gradient schemes for two-phase flow in heterogeneous porous media and Richards equation, ZAMM - Journal of Applied Mathematics and Mechanics, 94, 7-8, pp. 560-585, 2014.

[18] Faille, I., Fumagalli, A., Jaffré, J., Robert, J. Reduced models for flow in porous media containing faults with discretization using hybrid finite volume schemes, Computational Geosciences, 20,2, pp. 317-339, 2016.

[19] Flauraud, E., Nataf, F., Faille, I., Masson, R. Domain Decomposition for an asymptotic geological fault modeling, Comptes Rendus à l'académie des Sciences, Mécanique, 331, pp 849-855, 2003.

[20] Formaggia, L., Fumagalli, A., Scotti, A., Ruffo, P.: A reduced model for Darcy's problem in networks of fractures. ESAIM Mathematical Modelling and Numerical Analysis 48,4, pp. 1089$1116,2014$.

[21] Fumagalli, A., Scotti, A., An efficient XFEM approximation of Darcy flows in fractured porous media, Oil and Gas Sciences and Technologies - Revue d'IFP Energies Nouvelles 69,4, pp. 555-564, 2014 .

[22] Mayya Groza, Modélisation et discrétisation des écoulements diphasiques en milieux poreux avec réseaux de fractures discrètes, Thèse de Doctorat de l'Université Nice Sophia Antipolis, 2016, https://tel.archives-ouvertes.fr/tel-01466743/document

[23] Hajibeygi, H., Karvounis, D. and Jenny, P., A Hierarchical Fracture Model for the Iterative Multiscale Finite Volume Method, J. Comput.Phys. 230, 24, pp. 8729-8743, 2011.

[24] Hoteit, H., Firoozabadi, A. Numerical modeling of two-phase flow in heterogeneous permeable media with different capillarity pressures. Advanced Water Resources 31, pp. 56-73, 2008.

[25] Karimi-Fard, M., Durlovski, L.J., Aziz, K. An efficient discrete-fracture model applicable for general-purpose reservoir simulators, SPE journal, june 2004.

[26] Jaffré, J., Martin, V., Roberts, J. E. Modeling fractures and barriers as interfaces for flow in porous media, SIAM J. Sci. Comput. 26,5, pp. 1667-1691, 2005.

[27] Jaffré, J., Mnejja, M., Roberts, J. E., A discrete fracture model for two-phase flow with matrixfracture interaction, Procedia Computer Science 4, pp. 967-973 (2011)

[28] Lacroix, S. and Vassilevski, Y. V. and Wheeler, M. F.: Decoupling preconditioners in the Implicit Parallel Accurate Reservoir Simulator (IPARS). Numerical Linear Algebra with Applications, 8, pp. 537-549, 2001.

[29] Li, L. and Lee, S.H., Efficient Field-Scale Simulation of Black Oil in a Naturally Fractured Reservoir Through Discrete Fracture Networks and Homogenized Media, SPE Res Eval \& Eng 11 (4), pp. 750-758, 2008.

[30] Monteagudo, J.E.P. and Firoozabadi, A. Control-Volume Model for Simulation of Water Injection in Fractured Media: Incorporating Matrix Heterogeneity and Reservoir Wettability Effects, SPE Journal 12, 3, 2007.

[31] Reichenberger, V., Jakobs, H., Bastian, P., Helmig, R.: A mixed-dimensional finite volume method for multiphase flow in fractured porous media. Adv. Water Resources 29, 7, pp. 1020$1036,2006$.

[32] Sahimi, M. Flow and transport in porous media and fractured rock: from classical methods to modern approaches. John Wiley \& Sons, 2011, ISBN: 978-3-527-40485-8 
[33] Sandve, T.H., Berre, I., Nordbotten, J.M. An efficient multi-point flux approximation method for Discrete Fracture-Matrix simulations, JCP 231 pp. 3784-3800, 2012.

[34] Singhal, B.B.S. and Gupta, R.P. Applied Hydrogeology of Fractured Rocks, Springer Netherlands, 2010, doi: 10.1007/978-90-481-8799-7

[35] Tunc, X., Faille, I., Gallouët, T., Cacas, M.C., Havé, P. A model for conductive faults with non matching grids, Comp. Geosciences, 16, pp. 277-296, 2012. 